\title{
La identidad asexual: de la masculinización social a las redes sociales virtuales
}

\author{
The asexual identity: from the culture of masculinity towards \\ the social networks
}

\author{
Ma Teresa López Ruiz \\ tlrsoc@hotmail.com (ESPAÑA)
}

Recibido: 04.05.2015

Aceptado: 29.06 .2015

\section{RESUMEN}

En el marco de una tesis doctoral a propósito de la aparición, en la escena social, de la nueva identidad asexual, el presente trabajo indaga en los principales factores socio-culturales que pueden haber incidido en su desarrollo. Una identidad que se elabora intersubjetivamente ante nuestros ojos en el seno de una comunidad virtual y a la que, por tanto, no le pueden ser ajenos factores como las posibilidades que ofrecen actualmente las tecnologías de la información y la comunicación. Las nuevas redes sociales comienzan así a mostrarse, no sólo como instrumentos útiles para impulsar algunos cambios en las políticas públicas, o no sólo como generadoras de nuevas formas de comunicación, conocimiento y saber, sino como espacios donde se elaboran y se transmiten cambios, incluso en los aspectos que parecerían más íntimos del ser humano, como la propia sexualidad, en cuya normatividad ha enraizado siempre el deber ser- esto es, la identidad- de mujeres y hombres.

\section{PALABRAS CLAVE}

Asexualidad, cultura, identidad, masculinización social, redes sociales.

\begin{abstract}
Under the frame of a doctoral thesis, regarding the appearing of the asexual identity in the social scene, this work investigates the main cultural factors that could have contributed to its start and development. An identity under construc-
\end{abstract}


tion, that it's being produced here and now, in the bosom of a virtual community, and that can't be unconnected with the opportunities that offer the Information and Communications Technologies. The social networks start to seem, this way, not only as a useful resource to get changes in the public politics, and not only as a key to find new forms of communication and knowledge, but as spaces in which can be generated and disseminated changes, even in the more intimate and personal aspects of the human being, as the sexuality, in whose normativeness has always taken root the "must-be" (that is the identity) of men and women.

\section{KEY WORDS}

Asexuality, culture, identity, social contract, social networks.

\section{INTRODUCCIÓN}

Desde que en noviembre de 1859 se publicara la primera edición de El Origen de las Especies, la teoría de la selección natural -inspirada por la obra de Malthus- sirvió para inundar todos los órdenes de la existencia humana con la idea de la lucha por la supervivencia y el éxito del "más apto", sin que hayamos acabado de tener nunca muy claro en qué consiste tal cosa y cómo podemos saber quién es exactamente el más apto, excepto por la tautológica señal de que es el que sobrevive.

En lo que a sexualidad se refiere, la idea de la selección natural llegó a concretarse en la archiconocida imagen de dos ejemplares macho de alguna especie, midiéndose y luchando por su derecho a aparearse, lo que nos enseñaba lo que en cuestión de sexo era lo natural y también, por exclusión, lo que no lo era: en primer lugar, que lo natural en los machos era luchar entre sí (competitividad) y dividirse entre vencedores y vencidos (jerarquización, desigualdad); en segundo lugar, que por lo que había que luchar era, naturalmente, por el derecho de aparearse con una hembra, con el fin de que sólo uno de los machos, pero uno, se reprodujera (sexualidad heterosexual, selectiva, reproductiva, coitocéntrica) y finalmente, en tercer lugar, que era justamente esa lucha protagonizada en exclusiva por los machos (androcentrismo) la que llevaba nada menos que el peso y la responsabilidad de la evolución de la especie sobre sus espaldas (androcentrismo + competitividad + desigualdad + sexualidad heterosexual, selectiva, reproductiva, coitocéntrica $=$ progreso).

De ese modo sucinto, en la imagen más difundida y reduccionista de la selección natural, encontraba la modernización su justificación ideológica más acabada: una imagen en la que se condensaban todas las características que debían cumplir sus habitantes en el nombre del progreso, muy especialmente los hombres, si bien es cierto que las décadas posteriores vieron extenderse esa visión de lo que un ser humano debía ser a todas las sexualidades y todos los géneros: para contar en sociedad, todos ellos han debido demostrar su deseo de 
"normalizarse", que siempre ha querido decir heterosexualizarse, masculinizarse (Aguinaga, 2004), es decir adoptar ese grupo de valores que originalmente habían sido asignados exclusivamente a los hombres.

Desde esta perspectiva, el proceso de modernización podría ser observado como el proceso de masculinización social, esto es el proceso de convergencia social en torno a un conjunto concreto y funcional de valores, sancionados por la ciencia, y que actualmente parecería seguir su curso ciego y acelerado, en lo que ha venido a llamarse proceso de hiper-modernización.

$\mathrm{Y}$, sin embargo, a pesar de dicha aceleración y ceguera a otras realidades, hace tiempo que el mundo ha comenzado, a la vez, a ser otro (si es que alguna vez dejó de serlo) emprendiendo otros caminos en los que se han ido transformando las creencias, las necesidades y los hábitos que, hoy como ayer, también están comenzando a ser sancionados, aún lentamente, por la ciencia.

Como ejemplo, citemos que los avances científicos han permitido al género humano aprender a reproducir ejemplares de algunas especies, sin necesidad de reunir ADN masculino y femenino, es decir que ha conseguido clonar seres vivos, y con ello ha recordado, de forma visible y directa, lo que la existencia de seres sexuados en la naturaleza añadía realmente a la reproducción, que no es sino lo que en la clonación está ausente, esto es la diversidad y la variabilidad genética.

Muy brevemente, digamos que una especie en la que todos sus miembros resultan idénticos, es por ello mismo una especie en la que todos los individuos se ven afectados por igual ante una enfermedad o cualquier tipo de agente externo. No hay individuos mejor o peor preparados para afrontar cualquier determinada eventualidad. Si mueren, es más probable que mueran todos y que la especie desaparezca.

Desde ese punto de vista, la enorme variabilidad genética que ofrece, por su parte, la reproducción sexual, parece no tener precio: cada nuevo ser es único ${ }^{1}$, garantizando una diversidad que, en función de los cambios impredecibles en el entorno, podría acabar salvando a la especie. Pero la diversidad conlleva aún otra ventaja añadida: limita la competencia entre individuos, ya que al tener cada uno de ellos características ligeramente distintas, cada cual buscará satisfacer sus necesidades en nichos igualmente diferentes.

Las antedichas no son, aunque pudieran parecerlo, dos tesis opuestas sobre la evolución o la supervivencia en la naturaleza (la primera centrada en la idea de la competencia y la segunda en la de la diversidad) sino dos diferentes formas de mirar una sola teoría: la importancia de la diversidad ya estaba presente en Darwin. De lo que se trata es de a cuál de sus diferentes aspectos se decide, en

${ }^{1}$ Lo que nos obliga, directamente y desde el principio, al reconocimiento de que si hay algo que no existe en la naturaleza es, justamente, aquello de lo que se va tratar, es decir, la identidad, que es una construcción social. Butler (2007) por ejemplo, prefiere hablar por ello de identificaciones en lugar de identidades, que surgen de la necesidad de establecer semejanzas que hagan de puente con el prójimo, esto es que establezcan solidaridades grupales, pero cuyo inevitable reverso es determinar, a su vez, nuevas divisiones (e insolidaridades) con otros diferentes -pero igualmente- prójimos. 
una sociedad y momento determinados, y de manera más o menos consciente -aún desde el propio ámbito científico- prestar atención. Y la diversidad es hoy y aquí un concepto importante, que ha ido ganando terreno al de la competencia, como lo es el de la igualdad -frente a los supuestos "derechos naturales" de los "más aptos"- y como lo es también, inmersos como estamos en la sociedad del riesgo (Beck, 2002), la preocupación por la mera supervivencia en detrimento de fantasías evolutivas o determinados tipos de ambiciones de progreso que no en todos sus aspectos han parecido, finalmente, llegar a cumplir sus promesas.

La fatiga del dogma pro-masculinización, pro-sexual y pro-competitivo ${ }^{2}$ se ha ido sustanciando en múltiples formas de contestación, desde los ámbitos más públicos a los más íntimos. También las identidades sexuales construidas alrededor de tales principios se han ido poniendo en cuestión. Mujeres, pero también hombres heterosexuales que rechazan continuar encerrados en los cánones tradicionales de género (Stoltenberg, 2000); lesbianas y gays, que se niegan a vivir bajo las reglas de sus respectivos catecismos identitarios (Guasch, 2000; Preciado, 2011); intersexuales, personas transgénero, de género dual o neutro, que reclaman un espacio, material y simbólico, capaz de acoger unas realidades personales múltiples y plurales, sin acomodo en la configuración binaria de los géneros (Nieto, 2008), todos ellos conforman un universo postidentitario cada vez más poblado, en el que lo vital es el reconocimiento de la diversidad de la realidad humana, y del valor idéntico de cada una de las formas en las que puede llegar a expresarse esa realidad.

El movimiento para la visibilización y defensa de la asexualidad como una nueva identidad, ha venido a sumarse a este nuevo universo. Al igual que otros movimientos de liberación sexual, ha evolucionado desde lo que podríamos llamar un "programa fuerte" centrado en su propio "catecismo" asexual, hacia sus propias formas de contestación internas y hacia un proyecto más abierto e inclusivo, que acoge en su seno una multiplicidad de preferencias y realidades personales.

En cuanto sigue, se intentará explicar muy brevemente, en primer lugar, en qué consiste la identidad asexual y cómo su elaboración responde, en primera instancia, a la necesidad de construir una respuesta colectiva para explicarse, a uno mismo y a los demás, unos deseos y una forma de entender el mundo y las relaciones, que no coinciden con los sobre-entendidos habituales más generales respecto a la sexualidad, lo que desde la adolescencia acarrea no pocos choques, incredulidad o formas de desprecio, tanto en sus círculos sociales más inmediatos, como a la hora de poder plantearse la formación de una familia o la vida en pareja. Su emergencia sólo se entiende en relación -de oposición- a las definiciones sociales (elaboradas desde la heterosexualidad, pero también hoy desde la cishomonormatividad) respecto a lo que se considera "normal" o "anormal" en el ámbito sexual.

En segundo lugar, y en el entendimiento de que esas definiciones relativas a la sexualidad, son construcciones sociales, ancladas en los presupuestos

${ }^{2}$ Parafraseando a Echeverría, quien habla de la actual "fatiga del dogma pro-capitalista". 
culturales dominantes en un lugar y momento determinados, se analiza cuáles son entonces los concretos factores socio-culturales que pueden encontrarse más comprometidos en el desarrollo de la identidad asexual. Encontraremos que dichos factores son justamente aquéllos más relacionados con el proceso de masculinización social, sus tensiones y contradicciones, lejos de otras tesis y otras lógicas que han visto en la asexualidad, ya sea el resultado de un "excesivo" empoderamiento actual de las mujeres -que vendría a poner en crisis la identidad de los hombres- (Rosin, 2012), una consecuencia de la permisividad social respecto a las formas de ocio de los y las jóvenes -que banalizaría las relaciones sexuales -(Freitas, 2013), o la penúltima señal de un individualismo llevado al límite -por el que ya no se desearía la cercanía del prójimo ni siquiera para satisfacer necesidades afectivas o sexuales-(Spengler, 2012 ${ }^{3}$ ).

En su lugar, el proceso de elaboración de la identidad asexual se va a entender aquí imbricado en su relación -y coincidencia en el tiempo- con otros procesos más amplios, tales como:

A) Un clima social más o menos difuso, de apatía o cansancio respecto al significado -y frecuencia- de las relaciones sexuales, que podríamos denominar post-sexualismo, y que aparece de manera especialmente llamativa -y significativa- en aquellos países que tradicionalmente fueron considerados las "locomotoras" de la sociedad industrial, y que a algunos autores, como el propio Goldman, les ha llevado a la preocupación por el "futuro asexual de América" (Spengler, 2012, op. cit.) Reino Unido (Spiegelhalter, 2015) o Alemania (Inst. Rheingold, 2012), aunque en ninguno de tales países se dé un fenómeno tan llamativo como el de los llamados "hombres herbívoros" y los "hikikomori" en Japón (Ushikubo, 2008; Charlebois, 2013).

B) Un conjunto de procesos post-identitarios, a los que ya se ha hecho alusión, de contestación respecto a las identidades -homo y hetero- sexuales tradicionales y que han dado lugar a los fenómenos queer y transgénero (también, por ejemplo, en Butler, 2002, 2007).

C) Un contexto más general de des-afiliación social con respecto a otros valores e instituciones, que afecta desde los ámbitos más íntimos (como la paulatina pérdida del valor de la sangre a la hora de construir lazos familiares) hasta los más públicos o explícitamente políticos (como la desafección ante las formas tradicionales de la política) y en el que se origina una conciencia compartida sobre la necesidad de reformular los códigos y definiciones de la realidad, de ir des-aprendiendo lo aprendido; de emprender, de alguna forma, lo que Touraine (2005) definió como el proyecto de recomposición de lo social y de la experiencia personal, y en el que tienen cabida, por tanto, cada una de las formas en las que esa experiencia personal pueda llegar a expresarse, incluidas todas aquellas que se auto-inscriben dentro del paraguas de la asexualidad.

\footnotetext{
${ }^{3}$ Spengler es el pseudónimo con el que firma, en algunas ocasiones, David P. Goldman.
} 
Un espíritu, en definitiva, paralelo y opuesto a la hiper-modernización, de des-masculinización social, que se potencia, diversifica y difunde mejor allí donde los cuerpos y los roles asignados a cada individuo en sociedad, pueden estar más ausentes que en ninguna otra parte, es decir, en el espacio virtual. Dicho espacio, y sus relaciones con la comunidad asexual, en tanto que espacio de re-codificación cultural, des-situado y des-encarnado, constituirá, en tercer y último lugar, nuestro punto de destino. Todo ello, en el empeño de contribuir a la comprensión de lo que es y también, tal vez, sobre todo, de lo que no es la identidad asexual, y en el bien entendido de que, como se detallará unas páginas más adelante, cuando se habla de masculinización, se estará haciendo siempre referencia a la cultura de una sociedad, y nunca a características de la personalidad o individuales, ya sea de mujeres, hombres, personas de género dual, indeterminado o neutro. De lo que se habla es de sexualización, división, competitivización y, en suma, de aquellos rasgos que, como conjunto, hasta hoy han sido entendidos como darwinización social.

\section{ORIENTACIÓN, CONDUCTA E IDENTIDAD ASEXUAL.}

La comunidad asexual es un colectivo de personas que gana cada vez más adeptos en el mundo y que se han organizado para defender la asexualidad como una "cuarta orientación sexual". Actualmente, el número de personas que se identifican como asexuales aumenta cada día, y su principal plataforma virtual, la Asexuality Visibilitity and Education Network (AVEN, www.asexuality. org) fundada en 2001 en San Francisco por el joven estadounidense David Jay, cuenta con más de 110.000 integrantes que se comunican en 16 idiomas. Una de esas 16 áreas idiomáticas diferenciadas es la red para el área de habla hispana (Red para la Educación y la Vizibilización de la Asexualidad, REVA) más joven que su antecesora (2006) y que en la actualidad reúne a unas 3.500 personas. Se trata de un movimiento en el que, en conjunto, los hombres y las mujeres se encuentran representados prácticamente al 50\% y que está liderado -e integrado en su mayor parte, aunque no sólo- por jóvenes.

El colectivo define la asexualidad como la falta de atracción sexual por personas del mismo o distinto sexo, de manera estable en el tiempo y no como consecuencia de una enfermedad o cualquier otro impedimento. Son personas mental y físicamente sanas que, sencillamente, dicen no sentir ninguna necesidad ni interés por mantener relaciones sexuales, lo que no quiere decir que muchos de ellos no deseen pareja, otro tipo de intimidad con otra persona o que no sean capaces de enamorarse.

Los objetivos que persiguen son la visibilización y normalización de su orientación sexual, que las instituciones la integren y la reconozcan; trabajar para que la educación sexual recoja toda la diversidad de orientaciones sexuales, incluyendo la propia, y que la falta de atracción sexual deje de estar incluida en los manuales de psiquiatría como trastorno o disfunción mental. Las presiones 
en este sentido han dado ya algunos frutos y la última versión del Manual Diagnóstico y Estadístico de los Trastornos Mentales (DSM-5) presentado por la Asociación Americana de Psiquiatría el 18 de mayo de 2013, ha eliminado de su listado el "Trastorno por Aversión al Sexo" que sí recogían ediciones anteriores".

El paralelismo con los movimientos LGBT tanto en sus inicios como en cuanto a la evolución de las demandas, deseos y expectativas -así como las semejanzas entre sus discursos y los postulados queer- parecen importantes, lo que tal vez ayude a alejar la idea de asexualidad de otros conceptos tales como la abstinencia o la castidad, generalmente asociadas a algún tipo de fe religiosa: la mayor parte de los individuos asexuales se consideran ateos y para ellos la asexualidad no es algo que se elige voluntariamente sino algo que se es, tal y como otras personas se consideran homosexuales, heterosexuales o bisexuales.

Aunque se va a respetar el término asexualidad, por ser el comúnmente utilizado $^{5}$, la comunidad asexual acoge hoy en su seno otro tipo de sensibilidades y preferencias, que no encajan bien dentro de los estrechos límites de la definición de asexualidad, pero que tampoco se encuentran cómodas dentro del marco de la normatividad coitocéntrica habitual. Una diversidad que el propio colectivo resume y clasifica del modo siguiente:

${ }^{4}$ A cambio, el DSM-5 propone ahora el Trastorno Hipersexual (THS) como nuevo diagnóstico, asociado a otras dolencias como el Trastorno Obsesivo Compulsivo, Trastornos de Ansiedad, Trastornos Depresivos o Trastorno por Déficit de Atención e Hiperactividad. Las diferentes versiones del DSM han sido siempre controvertidas, pero nunca tan fuertemente contestadas públicamente como en esta última ocasión, al punto de que, pocos días antes de su presentación oficial, el Instituto Nacional de Salud Mental (NIMH) dependiente del gobierno estadounidense, anunciaba que comenzaría a financiar con carácter prioritario investigaciones que no utilizaran la clasificación DSM, mientras por otro lado, el "Comité Internacional de Respuesta al DSM-5" formado por representantes de varias organizaciones de Salud Mental y apoyado por la Asociación Americana de Psicología- presentaba una "Declaración pública de Preocupación por la próxima publicación del DSM-5" con el fin de advertir acerca de sus limitaciones, tanto a los profesionales de la psicología, como a los pacientes y los medios de comunicación. Todo ello puede verse en el sitio web del Consejo General de la Psicología de España: http://www.infocop.es/view_article. asp?id=4564. Por su parte, la Organización Mundial de la Salud recomienda la utilización de la clasificación del CIE (en inglés, IDC), cuya última versión data de 1990 (se espera la aprobación de su revisión para 2018) y, lo que tal vez resulte más significativo, cuya mencionada aprobación depende de los Ministros de Salud de los países integrados en la OMS. La diversidad sexual humana queda así encerrada, etiquetada y patologizada en clasificaciones afectadas por intereses, ya sean corporativos o expresamente políticos, bajo el barniz de la cientificidad, lo que afecta, a su vez, a aquello que la sociedad cree, y a aquello que los propios afectados llegan a creer, respecto de sus sentimientos, experiencias vitales y, en definitiva, sus realidades sexuales (en un proceso de doble dirección que, a su vez, determina las respuestas).

5 "Ace" es también un término muy utilizado y el preferido por muchos participantes, constituyendo el equivalente a "gay" o "straight" para homosexuales y heterosexuales. Surge del acortamiento fonético en inglés de la palabra "asexual", que a la vez hace referencia al "as" como "el mejor" y que ha dado lugar a un juego de auto-definiciones relacionado con los juegos de cartas, por el cual un "as de picas" es un asexual a-romántico y un "as de corazones" un asexual romántico; mientras el as de diamantes representa la demi-sexualidad y el de tréboles, lo Grey-A. 
- Asexuales: personas que no sienten ninguna necesidad ni interés por mantener relaciones sexuales.

- Autosexuales: personas que sí experimentan sensaciones sexuales, pero no sienten ninguna necesidad de compartirlas con otras personas.

- Demisexuales y Grey-A: situados en una "zona gris" donde las relaciones sexuales son esporádicas, dependen de la conexión emocional entre las partes, o bien se encuentran centradas en la "sensualidad" corporal antes que en la "genitalidad"6.

Para facilitar una visualización gráfica, utilizan el diagrama de Storms (1980), dentro del cual los diferentes grados o formas de asexualidad quedarían representados como un cuarto sub-universo, a añadir a los de la homo, la hetero y la bi-sexualidad, y que pueden encontrarse más cerca o más lejos de cada una de ellas en función de los sentimientos y experiencias de cada cuál.

Fig. 1. Diagrama de Storms.

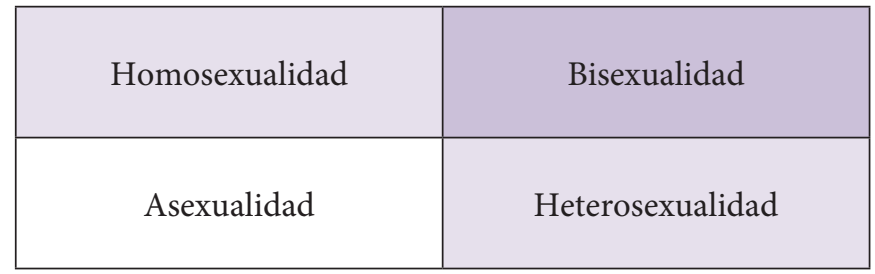

Fuente: www.asexuality.org

Hay que decir que la identificación con la asexualidad no siempre se corresponde con el comportamiento sexual real: al igual que existen personas que no han mantenido nunca relaciones sexuales, o bien las han mantenido en algún momento, pero por alguna razón han dejado de hacerlo y no por ello se autodefinen como asexuales, del mismo modo el hecho de que una persona se sienta integrada dentro de la definición de asexualidad no implica necesariamente que no haya mantenido nunca relaciones sexuales o que no esté dispuesto a mantenerlas en algún momento, ya sea por deseo de experimentar, por necesidad de sentirse "como los demás", porque tiene una pareja sexual y no desea perderla, etc. En la conducta sexual de los individuos influyen diversos y muy complejos factores. En lo que a asexualidad se refiere, Poston \& Baumle (2010), encontraron notables diferencias entre deseo, conducta e identidad asexual, en una investigación basada en datos de la población estadounidense:

${ }^{6}$ Una descripción de cómo las personas pueden establecer vínculos sensuales podemos encontrarlo, por ejemplo, en el estudio de la socialidad huaorani de la Amazonia ecuatoriana, en una cultura donde las relaciones sexuales se encuentran dirigidas específicamente a la reproducción y que no entiende "a los hombres y a las mujeres como clases de personas constituidas por y a través del deseo sexual (...)". (Rival, Slater y Miller, en Nieto (Ed.) (2003). Lo que nos lleva a la constatación de una aparente paradoja sobre el colectivo asexual, que se resiste a aceptar que sea el deseo sexual lo que defina a las personas y a sus relaciones íntimas, mientras él mismo se encuentra obligado a definirse en esos términos. 
- Conducta (no habían mantenido nunca relaciones sexuales): $6 \%$ de los hombres y $5 \%$ de las mujeres.

- Deseo (no habían deseado nunca relaciones sexuales): 0,7\% de los hombres y $0,8 \%$ de las mujeres.

- Identidad (se identificaban como asexuales): 3,9\% de los hombres, 3,8\% de las mujeres.

Por otro lado, el diagrama de Storms no recoge la pluralidad de inclinaciones románticas que, con independencia de su identificación con la asexualidad, pueden sentir quienes se autodefinen como asexuales y que pueden dividirse a su vez en a-románticos, homo, hetero, bi o pan-románticos, lo que da lugar a un universo plural de preferencias y relaciones, en las que parecen diluirse las fronteras entre el amor y la amistad. En este sentido, la identidad asexual "abre el campo de las relaciones (...) Hay muchas formas en las que los seres humanos pueden atraerse: existen la atracción emocional, la atracción intelectual, la afectiva... existe también una cosa que se llama atracción estética, etc. Miles de formas de atraernos o relacionarnos los unos con los otros". 7

Todas esas diferentes formas de vivir la asexualidad comparten una misma idea: la de la falsedad de la sexualidad como una necesidad natural, universal y constante. "El sexo se lo venden a uno como una necesidad humana, como el hecho de comer, pero no es así. Si no comes durante un mes, puedes morir, pero si no tienes sexo durante un mes, dos meses o durante un año ¿qué pasa? No pasa absolutamente nada". ${ }^{8}$

Dicho de otro modo, la asexualidad se construye como identidad colectiva frente a la sexualización social: lo que las personas asexuales tienen en común es que todos ellos han optado por dejar de identificarse activamente con la sexualidad "como marco social dominante para pensar en todo, desde el placer o el atractivo a la intimidad". . Por lo tanto, una persona asexual es, sencillamente, alguien que utiliza el término asexual para describirse a sí mismo ${ }^{10}$. "La etiqueta "asexual" sólo puede ser aplicada internamente, nadie tiene el poder de crear un conjunto de criterios que determinen quién es o no es asexual" 11 . El deseo de identificación proviene de una postura común en relación con las ideas culturalmente dominantes acerca de la sexualidad. Y es esa posición social común lo único que otorga carácter de unidad, de comunidad, a todas las personas asexuales.

7 Declaraciones de Johanna Villamil, Directora de REVA, en entrevista emitida en Radio Diversia el 1 de septiembre de 2012.

8 Johanna Villamil, en entrevista citada.

9 http://www.asexuality.org/wiki/index.php?title=Collective_identity_model

10 Tratando en su caso de transgenerismo y transexualismo, de forma muy similar escribe Nieto sobre la identidad de género "como autorreferente personalizado, emic. Sin sobreimposiciones del signo que sean de contenido etic: limitadoras, restrictivas o excluyentes de la libertad" del individuo. Y esa identidad de género, auto-definida, será la que determine, a su vez, la orientación sexual "hetero, homo, bi o asexual" (Nieto, 2008: 411-412).

11 http://www.asexuality.org/wiki/index.php?title=Collective_identity_model 
Recordando a Bordieu (1997) podríamos decir que una persona asexual es alguien con todas sus capacidades sexuales intactas, pero para quien el sexo no significa nada importante: ha dejado de formar parte, de algún modo, del capital simbólico que uno necesita para caminar -y ser feliz- por este mundo. Una más de entre las muchas actividades que un individuo puede o no practicar, al igual que cualquier otra actividad como, por ejemplo, el paracaidismo ( "Do you have hypoactive skydiving disorder?”, en Bogaert, 2012, 105-115).

La expansión y organización de la comunidad asexual implica, en definitiva, una nueva manera de suspender las normatividades sexuales: si los movimientos de liberación sexual cuestionaron antes que ellos el "con quién" del sexo (la heteronormatividad) hoy es el turno del "cómo" de las relaciones más íntimas entre dos personas, que ya no tienen por qué ser, además, necesariamente coitocéntricas. Una nueva forma de contestación a las identidades, presupuestos y creencias modernas sobre la sexualidad.

Una contestación, sin embargo, inscrita ella misma en aquello que niega: si lo que se defiende es que la sexualidad debe dejar de nuclear las relaciones más íntimas entre las personas, no se entiende bien que se utilice el término "orientación" como algo centrado exclusivamente en el sexo (o en este caso, en su ausencia). Si orientación significa hacia dónde se siente inclinado un individuo, es decir, por qué clase de personas se siente más atraído, tanto da si esa atracción es atracción sexual o exclusivamente romántica, aunque dicha atracción, como alguno de los representantes del propio colectivo nos apuntaba unas líneas más arriba, se encuentre fundamentada en lo estético, lo emocional, lo intelectual, lo afectivo, etc. En dicho sentido, podemos decir que se trata de una disidencia respecto a la sexualidad, ella misma sexualizada, lo que no impide en absoluto comprender el empeño de la comunidad asexual; por el contrario, de lo que nos habla, tal vez, es de esa necesidad de elaborar "un nuevo lenguaje, una nueva concepción de la vida personal y de su diversificación, que amplíe el horizonte -atendiendo menos exclusivamente al sexo o al género- y que vaya edificándose de abajo arriba, y no desde arriba hacia abajo" de la que nos advertía Plummer (en Osborne y Guasch, 2003), convencido de que aquello que une a todos los diferentes "grupos de intimidad", sexuales, asexuales o demisexuales, hetero, homo, bi, inter, trans, queer, románticos o a-románticos, es la necesidad de aprender a vivir y a reconocerse en un mundo plural y en permanente conflicto.

La identidad asexual es una contra-identidad más, que surge de experiencias personales reales y concretas de exclusión, de burla, de coerción, de soledad ${ }^{12}$, de patologización y medicalización de las preferencias sexuales, sufridas dentro

12 Por razones de espacio, como muestra de la clase de experiencias de las que se habla, se anima al lector a visitar los foros de la comunidad asexual, en cualquiera de sus idiomas. En el caso del área de habla hispana, baste decir que las palabras "depresión", "suicidio" o "morir" son recurrentes, a pesar de los esfuerzos de los administradores -en el caso del suicidio- por desterrarlas. En sus discursos, los citados términos se encuentran asociados a las imágenes que de la asexualidad ellos mismos reciben del exterior, no sólo, pero muy especialmente, desde el campo de la psiquiatría, desde donde se patologizan -y medican- sus preferencias. 
de una sociedad que no acaba de alcanzar conciencia de esa pluralidad y de esa necesidad de aprender a reconocerse, y a reconocer, en definitiva, que tal vez la única identidad que importa es “...la identidad en la democracia. Y la identidad en la democracia quiere decir identidad en la cultura, identidad en la libertad, identidad en la justicia, identidad en la piedad, identidad en la solidaridad, identidad en la verdad, identidad en la belleza. Esa es la verdadera identidad de los seres humanos. Lo demás es, en el peor sentido de la palabra, mala literatura". ${ }^{13}$

\section{DIMENSIONES CULTURALES Y ASEXUALIDAD.}

En el marco teórico de la acción humana como acción simbólica -esto es, como acción cuyos concretos significados se encuentran mediados por la cultura- lo que nos proponemos ahora es la identificación de los principales factores culturales que puedan haber facilitado el surgimiento y desarrollo actual de la identidad asexual. Ya se ha adelantado que, en primera instancia, se trata de una respuesta a la sexualización/sexualismo reinantes. De lo que se trata ahora es de verificar su relación con ese algo más amplio que hemos llamado masculinización, de la que la sexualización constituiría el primero de los requisitos.

Para ello, van a utilizarse las dimensiones culturales identificadas y operacionalizadas por Hofstede (Hofstede et al, 1998; Hofstede, 2001; Hofstede et al, 2010), cada una de las cuales está conformada, a su vez, por diferentes conjuntos de valores y cuyas relaciones con la sexualidad humana han sido ya abordadas por algunos investigadores españoles (Ubillos et al., 2000), quienes encontraron correlaciones significativas entre una mayor frecuencia de relaciones sexuales y un alto grado de aversión a la incertidumbre, mayores índices de individualismo, una escasa cultura del poder y una baja masculinización de las sociedades. Es el miedo a que nuestro universo conocido se derrumbe (esto es, la aversión a la incertidumbre) el primero de los factores culturales que parece empujarnos con mayor frecuencia que otros hacia las relaciones sexuales. Y esto ya es un dato importante.

De manera similar, podríamos preguntarnos si el mayor o menor desarrollo de la comunidad asexual puede estar relacionado con esas mismas dimensiones culturales. Los datos de los que disponemos sobre la comunidad asexual son los del total de integrantes de cada una de sus 16 plataformas (recuento de elaboración propia a 26 de diciembre de 2012), su crecimiento diferenciado a lo largo del último año (recuento de elaboración propia a 26 de diciembre de 2013) y las diferentes tasas de actividad en cada plataforma (número de mensajes

${ }^{13}$ Lledó, E. "El Lenguaje de la Identidad". Congreso de la Fundación Caballero Bonald bajo el título "Periodismo y Literatura" celebrado en Jerez de la Frontera (Cádiz - España) 17, 18 y 19 octubre 2007. Publicado por Revista Literaturas.com (marzo 2013) y disponible en: http://www. literaturas.info/Revista/2013/03/el-lenguaje-de-la-identidad-emilio-lledo/ 
totales, tanto en valores absolutos como respecto al número total de integrantes de cada comunidad). Las grandes diferencias encontradas para tales valores, entre los países en los que tiene presencia la comunidad virtual, nos servirán para observar si existe algún tipo de correlación entre el mayor o menor éxito del colectivo asexual y las puntuaciones de dichos países en cada una de las dimensiones culturales.

Pero para poder establecer tales comparaciones, existen algunos otros aspectos metodológicos que es necesario señalar. Hay que recordar que, tratándose de un análisis comparativo de factores culturales, existen variables no culturales que se deben controlar:

a) En primer lugar, y como es evidente, hay que tener en cuenta que cada país tiene un número muy distinto de habitantes, lo que influye por sí solo en el número de personas que pueden llegar a adscribirse a una comunidad virtual. Por ello, el número de integrantes de cada plataforma de AVEN se presenta en tasas por 100.000 habitantes (en datos de población del Banco Mundial, relativos a 2013).

b) En segundo lugar, la comunidad asexual es un tipo de comunidad muy específica, que es una comunidad online. Ello quiere decir que su número de integrantes se verá influenciando, igualmente, por el grado de accesibilidad, en cada país, a Internet y a las redes sociales virtuales. Con el fin de que los resultados no se vean distorsionados por dicho factor, las tasas de integrantes de la comunidad virtual han sido ponderadas en función del nivel de acceso de cada país a Internet y las redes sociales virtuales (en datos del Banco Mundial relativos a 2013), es decir, se ofrecen bajo el supuesto de que todos los individuos tienen igual acceso a Internet. ${ }^{14}$

c) Además de la mayor o menor facilidad de acceso a las redes sociales, la siguiente barrera de entrada, en una comunidad on-line, no puede ser sino idiomática. Un mismo individuo, que sepa desenvolverse en diferentes idiomas, puede estar participando a la vez en distintas plataformas, y nosotros podemos estar contabilizándolo no sólo una sino dos o más veces. Este es un punto difícil de controlar, en el que lo único que podemos hacer es tener en cuenta el peso de los nacionales de diferentes países, en las principales plataformas en las que su presencia es ya conocida: en la comunidad de habla hispana, el mayor peso corresponde a países como España, México y Argentina; en el área anglosajona hay que tener en cuenta, prioritariamente, a EEUU y Reino Unido y en menor medida, a los integrantes procedentes de Australia y Canadá ${ }^{15}$. Finalmente, en el caso de la República Checa, su plataforma es utilizada por

${ }^{14}$ Tanto en España como en Europa contamos con diversos ejemplos de investigaciones que han utilizado tales ponderaciones de variables, como los estudios sobre Internet y participación política en España, donde se comparan datos de diferentes comunidades autónomas (Anduiza et. al., 2010), o bien trabajos en los que se utiliza información de diferentes países, como los de la Fundación WageIndicator (Tijdens, K.G. y Van Klaveren, M., 2012). Un sitio web especialmente dedicado a la metodología de investigación online es el Web Survey Methodology (http://www. websm.org).

${ }^{15}$ http://www.asexuality.org/home/2008_stats.html 
los integrantes de la comunidad que residen en la República Eslovaca. El peso del porcentaje de usuarios de cada país en las tres plataformas citadas, así como las correspondientes puntuaciones para cada dimensión cultural, han sido igualmente ponderadas.

En lo que a las propias dimensiones culturales se refiere, es preciso señalar dos cuestiones fundamentales:

- Que las dimensiones culturales estarán siempre referidas a los rasgos característicos de una sociedad, y nunca a características de la personalidad o individuales. Cuando se hable, por ejemplo, del índice de "masculinización" social, o simplemente de "masculinidad" no nos estaremos refiriendo de ningún modo a los hombres, sino a la asunción general, por parte de una sociedad como conjunto, de los valores tradicionalmente asignados a la masculinidad, es decir, a una cultura en la que primen los valores del trabajo y de la competitividad por encima de otros, como la sociabilidad, el tiempo libre, la familia o los amigos.

- Que las dimensiones culturales sólo son susceptibles de puntuarse en términos relativos, es decir, de comparación entre sociedades y nunca como medidas pretendidamente objetivas o absolutas de valores (Hofstede et al., 2010). No existe una medida que nos permita decir, por ejemplo, que una sociedad es demasiado individualista o colectivista (en todo caso, habría que explicar en relación con qué fin tales valores nos resultan excesivos). Lo único que podremos decir al respecto es que una sociedad se muestra, en un momento determinado, más -o menos- individualista (o cortoplacista, o permisiva, o igualitarista) que otra u otras.

En el caso del análisis de las dimensiones culturales, la ponderación de resultados en la forma descrita, resulta especialmente necesaria, por una cuestión que no debe pasarnos desapercibida: el riesgo de sobre-dimensionar la importancia de algún factor cultural -especialmente el individualismo- en los estudios sobre participación online. De todos es sabido que en las sociedades más individualistas es donde más herramientas tecnológicas se utilizan, donde más televisores hay en los hogares, y también donde más teléfonos móviles y ordenadores existen por individuo (Hofstede et al., 2010). Por lo tanto, la posibilidad de errar el tiro, atribuyendo al individualismo una relación directa exagerada con cualquier variable dependiente asociada con el uso de Internet, en éste o en cualquier otro estudio (cuando lo que realmente están mostrando los resultados es que en determinados lugares Internet se utiliza con mayor facilidad que en otros) resulta demasiado grande como para poder pasarla por alto. La influencia del individualismo -o cualquier otro factor cultural- cuando existe, aparecerá de cualquier modo (como, de hecho, así ocurre en nuestro caso) cuando se ponderan los datos descritos, pero sus relaciones deben ser susceptibles de medirse en sus justos términos. A cambio de esta ventaja metodológica, lo que deberá tenerse presente en la investigación, es que la ponderación de resultados no sirve para otros fines, es decir no implica de ningún modo que los resultados puedan extrapolarse a la población offline. Cualquier resultado obtenido se encontrará referido a los estrictos límites del universo virtual que hayamos previamente definido. La investigación presente 
no intenta ir más allá: lo que se pretende es poder esbozar algunas claves sólidas para la comprensión de la identidad asexual, a través de la ventana que ofrece la propia existencia de la comunidad virtual, y que tal vez pueda servir a ulteriores investigaciones, con otros instrumentos de análisis o sobre universos poblaciones más amplios.

Finalmente, hay que considerar que, para el caso de algunos países, existen dificultades metodológicas añadidas, además de las dificultades de acceso a Internet, que no pueden ponderarse, ya que el acceso a determinados tipos concretos de información en la red, plataformas o comunidades online, puede verse restringido en determinados momentos en algunos lugares, o bien porque, sin tales restricciones, la configuración de los sitios web es radicalmente distinta a la del resto de países, no permitiendo verificar o establecer comparaciones entre las diferentes variables ${ }^{16}$. Por ello, en el apartado correspondiente, el análisis va a limitarse a 12 de las 16 plataformas de AVEN.

Por su parte, el caso de la comunidad de habla hispana (REVA) reviste para nosotros, por razones evidentes, un interés especial. En ella participa también población de muy diferentes países cuyos niveles de accesibilidad a las redes virtuales son también muy dispares, y su análisis va a efectuarse de manera independiente, como un caso particular dentro del más general de la comunidad AVEN. Ello va a permitir, además, disponer de una fuente de datos adicional con la que verificar o matizar las posibles relaciones que puedan encontrarse en el primer caso de estudio (el del conjunto de países en los que está presente AVEN). En este sentido, hay que subrayar que la plataforma REVA no ofrece datos directos sobre la nacionalidad de sus integrantes, por lo que los datos que van a utilizarse proceden de un cuestionario on-line elaborado al efecto (muestra autoseleccionada, obtenida en enero de 2012, $\mathrm{n}=100, \mathrm{~N}=2440$ ), y en el que el nivel de participación de cada nacionalidad ha sido ponderado igualmente en función del grado de acceso a Internet de cada uno de los países de referencia. Todo lo cual habrá de ser tenido en cuenta a la hora de interpretar correctamente los resultados.

Debemos insistir en que lo que buscamos no son las causas, biológicas, psicológicas o de ninguna otra índole que pudieran eventualmente dar cuenta de la asexualidad, cuya existencia, antigua y real, hemos dado por descontada. Desde los estudios de Alfred Kinsey (Kinsey et al., 1948, 1953) sabemos que en nuestras sociedades siempre ha existido un porcentaje de personas que no desean ni mantienen relaciones sexuales. Lo que nos preguntamos no es por qué razones existe la asexualidad sino por qué razones emerge hoy como identidad colectiva: ¿por qué se necesita visibilizar y poner un nombre a lo que sin tanta luz había existido desde siempre? ¿Por qué razones aumenta cada día que pasa el número de integrantes del colectivo asexual? ¿Cuáles son los significados de la

${ }^{16}$ Las diferencias referidas pueden observarse accediendo a cada una de las diferentes plataformas, desde la parte inferior de la página de inicio de la web www.asexuality.org. Los países en los que AVEN tiene presencia, pero no han sido incluidos en el estudio, son China, Israel, Rusia y Turquía.

EMPIRIA. Revista de Metodología de Ciencias Sociales. N. ${ }^{\text {32 }}$, septiembre-diciembre, 2015, pp. 129-168. ISSN: 1139-5737, DOI/empiria. 32.2015.15312 
sexualidad humana que están cambiando y en qué nuevas direcciones?

De manera muy sintética, el conjunto de hipótesis previas de las que se parte pueden resumirse del siguiente modo:

- Se está produciendo un nuevo cambio en el significado de la sexualidad, opuesto al sexualismo reinante (y una de cuyas más recientes expresiones es la denominada "cultura hook-up" (Freitas, 2013)). Dicho cambio devalúa el valor simbólico de las relaciones sexuales, subrayando su carácter ritual (obligación social).

- Si lo que pone en evidencia la identidad asexual, devaluándolo, es el carácter de rito social de la sexualidad (sea homo o heterosexual), la aparición de esta nueva identidad tendrá que ver con el valor actual de los ritos sociales, esto es: con la salud del contrato social (que en la moderna sociedad industrial, ha sido siempre socio-sexual (Pateman, 1995)).

- Las dimensiones culturales más relacionadas con la comunidad asexual habrán de ser entonces aquéllas que más se encuentren asociadas al declive actual de ese contrato y al "deber ser" de hombres y mujeres que se transforma, en la misma medida en la que han ido transformándose las instituciones modernas y su correspondiente conjunto de valores y creencias.

- En cualquier caso, el contrato social declina en el mismo momento y hora en el que, por su parte, la tecnología ofrece vías para salvar tiempos y espacios, facilitando la reunión de personas que de otro modo no podrían haberse comunicado. Las características de este nuevo ágora inesperado, deberán hallarse también en el núcleo de la pre-figuración de un nuevo "deber ser" (en el que la sexualidad -y las identidades sexuales de mujeres y hombres- tienen menor significación que en etapas anteriores) y para cuya aprehensión el estudio de la identidad asexual, en tanto que nos informa de las transformaciones en curso a partir de lo más íntimo de la experiencia, puede constituir un espacio de análisis privilegiado.

\subsection{La cultura del poder: power distance index (PDI).}

La primera de las dimensiones culturales que vamos a utilizar, nos informa acerca del grado en el que dentro de una sociedad las desigualdades y los privilegios que acompañan al poder son justificados o aceptados. No se trata de medir los niveles objetivos de desigualdad, sino de la forma en la que tales niveles son considerados por la población como algo normal, conveniente o apropiado.

Al igual que en otros ámbitos, en materia de sexo el poder ha de dejar constancia de que lo es, y es más fácil encontrar el pensamiento por el cual “... no se concibe un hombre poderoso sin un montón de amantes jóvenes y apetitosas"17. En el mundo de la familia y de la pareja, lo que se espera es

${ }^{17}$ La expresión se recoge literalmente de la aportación de un internauta que, bajo el Nick de "heartguitar", comenta la noticia aparecida en prensa sobre la condena a Silvio Berlusconi por el 
respeto (en el límite, obediencia) hacia el cabeza de familia y los episodios de violencia pueden darse con mayor frecuencia. Las relaciones más íntimas suelen reflejar la misma cultura desigualitaria y son más a menudo un ámbito en el que también se dirimen cuestiones de poder, de privilegios o status.

Toda vez que en la mayoría de los países considerados, se encuentra ya instalada una mayor cultura igualitaria entre los sexos, la primera pregunta que podríamos hacernos es si ha sido esa mayor igualdad la que ha conllevado un cambio en el significado de la sexualidad, es decir, si es posible que para algunos hombres -y mujeres- las relaciones hayan perdido algo de su encanto o de su gracia, o si quizás ha provocado un sentimiento de desplazamiento -o aburrimiento- en los hombres que afecta a las relaciones, tal y como ha sido señalado por algunos autores (Rosin, 2012). ¿Es eso lo que ha ocurrido? ¿Es en los países más igualitarios o, por el contrario, en aquellos donde existe una mayor cultura de la desigualdad, donde existe un mayor número de personas que se integran en la comunidad asexual?

\subsection{El Individualismo (IDV).}

El individualismo se define como el grado en el que dentro de una sociedad está consolidada la idea de que los derechos y deberes deben corresponder al individuo y no a los grupos sociales a los que pertenece (como la familia, el clan o la tribu). En los países más individualistas, las personas esperan que cada cual se ocupe únicamente de sí mismo y de sus familiares más cercanos; en los hijos se valora la personalidad independiente, proactiva y autónoma; la ocupación, los amigos, el lugar de residencia y la pareja pueden elegirse libremente, etc.

El individualismo es también universalista: si el valor de cada individuo no depende del valor del grupo de pertenencia, todos los individuos valen igual y tienen por tanto los mismos derechos y las mismas obligaciones.

A la cultura individualista se le han atribuido muchos de los males que aquejan a nuestras sociedades. En el ámbito de la pareja, la "guerra de los géneros” (Beck, Beck-Gernsheim, 2001) o el “amor líquido” (Bauman, 2005) son algunas de las expresiones con las que se ha apuntado hacia el individualismo como principal culpable del desinterés por el compromiso, las caídas en las tasas de nupcialidad y natalidad o de los altos índices de rupturas matrimoniales. ¿Cabría ver la identidad asexual bajo este prisma? ¿Se trata de un deseo de autonomía llevado al extremo? O, sin llegar a ese extremo ¿Se trata, mucho más sencillamente, de que la idea implícita del sujeto como autor y responsable de su propia vida, fomenta la elaboración de cualquier tipo de identidades, incluida la asexual? ¿Es en definitiva en los países más individualistas donde existe un mayor número de integrantes de la comunidad asexual?

caso "Ruby" el 25 de junio de 2013.

EMPIRIA. Revista de Metodología de Ciencias Sociales. N. ${ }^{\text {32 }}$, septiembre-diciembre, 2015, pp. 129-168. ISSN: 1139-5737, DOI/empiria. 32.2015.15312 


\subsection{Competitividad vs. calidad de vida: Masculinity vs. Femininity (MAS).}

Esta tercera dimensión hace referencia al grado en el que en una cultura prevalecen los valores tradicionalmente considerados masculinos o bien, por el contrario, aquellos otros más atribuibles a un hipotético universo femenino, es decir de un lado la competitividad, el valor, la fuerza, la audacia, el interés por el logro económico, frente a un polo opuesto en el que encontraríamos la cooperación, la prudencia, la sensibilidad, la empatía, la importancia de las relaciones con los demás o la preocupación por la calidad de vida. En un país con altos índices de masculinidad, la esfera pública o laboral y la esfera privada o familiar se encuentran muy separadas: lo importante es el trabajo y lo que se valora es la capacidad de cada persona para dedicarse en cuerpo y alma a su empresa o a su profesión. Por el contrario, en los países más femeninos, costaría entender que un profesional (hombre o mujer) no pidiera permiso para acudir, por ejemplo, a las reuniones periódicas con los profesores de sus hijos o que no cuidase de ellos en casa cuando estuviesen enfermos.

En un país masculino, las personas, los grupos, las organizaciones, compiten entre sí: lo que importa es ser el más listo, el más fuerte, el más rápido, el más atractivo (aun sólo en el terreno público) mientras en un país femenino la aspiración fundamental es poder llegar a cumplir con todo aquello a lo que uno está comprometido. Por lo que se refiere a la sexualidad, como era de esperar, también puede ser motivo de competición -simulada o real- o bien una cuestión enmarcada en la privacidad de cada cual. En las culturas masculinas el sexo es una hazaña, una prueba, no ya de poder, sino de masculinidad, mientras que en las femeninas, tanto para los hombres como para las mujeres, la sexualidad es relación, una de las muchas formas de sentirse en conexión con el semejante. La cultura masculina implica una mayor presencia del sexo en el espacio público (hiper-sexualización) y una mayor abundancia de simbolismos, chistes y sobreentendidos sexuales en las conversaciones.

En este punto es importante distinguir entre esta tercera dimensión y la primera de las mencionadas (PDI) pues en lo que a sexualidad se refiere, no se trata tanto de la expresión del poder, como del "deber ser" de cada cual, ya se trate de un hombre o de una mujer. Los hombres deben demostrar, no que son poderosos, sino que son hombres, y las mujeres, mujeres: la prueba de que uno efectivamente es lo que debe ser se dirime también, y sobre todo, en el ámbito de la sexualidad ${ }^{18}$.

Al separar las esferas laboral y familiar, en una sociedad masculina la conciliación suele resultar más difícil que en otras sociedades; los roles, los

${ }^{18}$ Igualmente, hay que subrayar que una sociedad masculina o femenina no significa nada parecido a una sociedad machista o feminista. Lo que podremos observar, en todo caso, serán diferentes formas de machismo o feminismo en cada una de ellas. Por ejemplo, en una sociedad masculina, las demandas del feminismo estarán más centradas en defender la igualdad en el ámbito público o laboral (igualdad de salarios, "techos de cristal") mientras en una sociedad femenina cabría esperar que se hablase más de conciliación y corresponsabilidad en el hogar. 
comportamientos pero, sobre todo -y mucho más relevante para esta dimensiónlas emociones y los sentimientos "permitidos" o "prohibidos" son diferentes para los hombres y las mujeres: los hombres han de ser rudos, competitivos, no lloran, mientras en una cultura más femenina las muestras de ternura, comprensión o solidaridad son algo perfectamente aceptable para ambos géneros.

En definitiva, tener que vivir o no para cumplir una misión "sagrada"(la laboral) separada de nuestras necesidades humanas básicas, mucho menos importantes - es decir, lo que podríamos resumir en el "vivir para trabajar" o "trabajar para vivir"- son dos opuestos que bien pueden caracterizar las diferencias entre los dos polos de la dimensión MAS/FEM (Hofstede et al., 2010:170), un rasgo que no puede dejar de recordarnos a la cultura japonesa, asociada como pocas al vivir para trabajar, a la importancia de la lealtad a la empresa y muy significativamente patria actual de los "hombres herbivoros" o los "hikikomori", así como en Europa, Alemania, cuyas tasas de integrantes de la comunidad asexual están entre las más altas junto a las de EEUU. Tampoco se nos escapa que esta dimensión nos ha hablado ya, como el individualismo, del "deber ser" de cada cuál. Es decir, si en el seno de una cultura individualista uno está obligado a auto- realizarse y desarrollar lo que es, la dimensión MAS es la que problematiza, en mayor o menor grado, qué es aquello que uno debe ser - un hombre o una mujer- con todas las consecuencias en los ámbitos de la sexualidad y el trabajo. Es, por tanto, la dimensión más relacionada con las identidades sexuales tradicionales. ¿Existe una correlación real entre el éxito del colectivo asexual y el conjunto de valores que hemos llamado "masculinos" o "femeninos", entre la aceptación más o menos plena de la cultura de la hipercompetitividad y la sexualización actual (puro síndrome de masculinidad (Hofstede et al., 1998)) y la fuerza o debilidad de la comunidad asexual?

\subsection{El miedo a los cambios: Uncertainty Avoidance Index (UAI).}

El índice de aversión a la incertidumbre se define como el grado en el que los miembros de una cultura se sienten amenazados ante situaciones inesperadas, desconocidas pero, sobre todo, ambiguas. No se trata tanto de un miedo al riesgo, como de una aversión a lo incierto: lo importante es saber que se está en "la verdad", en "la razón", en el camino "correcto"; saber y que se sepa con claridad lo que uno ha de hacer, a qué debe atenerse y qué debe esperar de los demás. En este sentido, en los países con altas puntuaciones en este índice, las normas, leyes y costumbres se defienden más que en otras sociedades, se convierten en un valor en sí mismas y se tornan más rígidas. El ideal clave es el de pureza: allí es donde cabe esperar una mayor intensidad de las reacciones sociales por cuestiones ideológicas, nacionalistas, étnicas o religiosas, una mayor resistencia a los avances tecnológicos y a los nuevos productos y, como no podía ser de otro modo, un rechazo también mayor a la desviación de la norma en materia sexual, es decir a cuestiones como el aborto, el divorcio o a cualquier 
tipo de relaciones que puedan amenazar las normatividades sexuales. Según la investigación citada (Ubillos et al., 2000) es precisamente en este tipo de sociedades donde la frecuencia de las relaciones sexuales suele resultar mayor, pues a menudo se convierten en un remedio contra la ansiedad y en una ocasión para comprobar que entre la pareja (o incluso en el interior de uno mismo) todo continúa "en orden".

En países con menores puntuaciones, la fe en verdades absolutas e intemporales es menor, las normas son sólo un medio para obtener un fin y se considera que son ellas las que deben adecuarse a las realidades sociales. Por lo tanto, tal vez cabría esperar un mayor número de personas dispuestas a identificarse como asexuales en aquellos países con mayor flexibilidad normativa y menor miedo a lo nuevo, a lo desconocido o lo ambiguo, en definitiva, a algo como el "amoeba lifestyle". ¿Es ello realmente así? ¿Ha sido el relajamiento progresivo de las normas con respecto a la sexualidad las que han permitido a algunos hombres y mujeres renegar, primero de la heteronormatividad, pero después también del coitocentrismo? ¿Hasta qué punto podemos decir que está relacionada esta cuarta dimensión cultural con la identidad asexual?

\subsection{El ahora o el futuro: Long-Term Orientation (LTO).}

Este quinto indicador se define como el grado en el que las sociedades están orientadas hacia el logro al corto o al largo plazo. En sociedades cortoplacistas la relación con la realidad social es sincrónica: lo que importa es el ahora, cumplir con lo que la sociedad requiere de nosotros en el momento actual, mantener las relaciones con aquellos que nos acompañan y necesitamos hoy y actuar de acuerdo a nuestras necesidades inmediatas sin pensar en el mañana. Por el contrario, en las culturas orientadas al largo plazo, la relación con la realidad social es diacrónica: lo que uno hace hoy y el tipo de relaciones que mantiene tienen que ver con lo que se desea o espera para el futuro, y se fomentan valores como la perseverancia y el ahorro. En este tipo de culturas, se considera que la vejez comienza a una edad más temprana y que se trata de una etapa más feliz y deseable que en las cortoplacistas; la verdadera plenitud no se asocia a la juventud, sino a etapas más avanzadas de la vida, por lo que podría pensarse que para algunos individuos la sexualidad pudiera perder su lugar nuclear a favor de otros aspectos relevantes de las relaciones de pareja. Pero para explicar mejor esta dimensión, es preciso añadir que, además, lo que se dirime en su seno es una orientación al corto o al largo plazo es este mundo y no en ningún otro. El sacrificio, la perseverancia y el ahorro son valorados en tanto que medios para alcanzar un futuro mejor aquí en la tierra, y no como valores absolutos a través de los cuales uno pueda garantizarse un buen lugar en el más allá. La dimensión LTO, conocida también como confucianismo, y cuya elaboración tiene su origen en la Encuesta China de Valores (CVS, Hofstede et al., 2010: 235-276) recoge un conjunto de consideraciones éticas que no se encuentran atravesadas por el pensamiento religioso a la manera occidental. No existen la 
verdad y el bien absolutos, y lo que cada cual debe o no debe hacer no depende de la tradición ni de verdades eternas, tanto como de las circunstancias y de las implicaciones prácticas del comportamiento para el futuro. Ello puede explicar que algunas culturas cortoplacistas puedan mostrar, paradójicamente, un menor pragmatismo y un mayor apego a la tradición en lo que se refiere a cuestiones sexuales, tal y como ocurre con el divorcio o el aborto, o incluso, tal vez, respecto a una sexualidad "monotemática" en la que, en no pocas ocasiones, la pareja implicada sólo trate de "cumplir" con el momento, en lugar de intentar satisfacer sus verdaderas necesidades afectivas y/o sexuales. Una cultura cortoplacista, en la que los jóvenes experimenten la urgencia de estar a la altura de los que requiera su grupo de referencia, sin cuestionar nada más, de hecho parecería llevarnos más bien a la "cultura hook-up" (Freitas, 2013, op.cit.) que a otro tipo de identidad, como la asexual. En cualquier caso, si existiera una correlación positiva entre la comunidad asexual y el cortoplacismo, ello podría estar indicando que esa identidad tiene mucho más que ver con los significados de la sexualidad en el momento actual: la pérdida de valor de las identidades sexuales se produciría entonces como consecuencia de las percepciones en torno a un aquí y ahora determinados.

\subsection{La cultura del ocio: la importancia del tiempo libre y de los amigos. Indulgence vs Restraint (IVR).}

Finalmente, este último indicador hace referencia al grado en el que una sociedad se muestra permisiva específicamente respecto a las actividades relativas al disfrute de la vida y el tiempo libre, o por el contrario se considera que tales aspectos deben estar socialmente pautados y controlados. En las sociedades con las más altas puntuaciones de este indicador, las personas suelen sentirse más felices y optimistas, tienen más hijos, perciben mayores niveles subjetivos de salud y bienestar y también un sentimiento de mayor libertad y control sobre la propia vida. La propensión a la diversión y al buen ánimo frente a las adversidades, así como el ser una persona sociable y tener muchos y buenos amigos, son aquí valores clave. La comunicación y el contacto personal o virtual- con los semejantes se dan con mayor frecuencia. Las normas respecto a la sexualidad son más flexibles y existe una mayor tolerancia hacia la promiscuidad o las relaciones sexuales ocasionales.

Por el contrario, unas formas de ocio más pautadas y menos libres, exigen por definición una espontaneidad menor, una mayor contención y un menor contacto gratuito con el semejante. En su forma extrema, un exceso de control sobre la sociabilidad y el disfrute del tiempo libre podría resultar en apatía, temor al prójimo y a las relaciones sociales, lo que no puede dejar de recordarnos a los jóvenes hikikomori como imagen extrema de una identidad y una vida casi exclusivamente virtuales.

En lo que concierne a esta última dimensión ¿cuál de sus dos extremos puede estar más relacionado con la identidad asexual? ¿Es acaso una de las 
consecuencias de esta especie de apatía juvenil, temerosa y recluida, o es por el contrario una identidad mucho más ligada a la imagen de los más sociables "hombres herbívoros", a la importancia de los amigos, el disfrute de la vida y el tiempo libre, por encima de la familia o de la pareja?

\subsection{Resultados}

Una vez calculadas las posibles correlaciones entre las puntuaciones de cada país en cada una de las dimensiones culturales, y sus diferencias en cuanto al número de integrantes de las diferentes plataformas virtuales (en tasas por 100.000 habitantes), así como con su crecimiento durante el último año y con las tasas de actividad en los diferentes foros, los resultados pueden resumirse del siguiente modo:

a) El número de integrantes: la dimensión MAS.

En lo que concierne a las diferencias en el número total de personas integradas en cada plataforma (y una vez efectuadas las ponderaciones tal y como se muestra en la Tabla 1), podemos decir que la dimensión que correlaciona de manera más significativa con el éxito de la comunidad asexual es la cultura de la masculinidad (dimensión MAS), es decir aquella a través de la cual la identidad de los individuos ha quedado siempre ligada a la normatividad sexual, la división del trabajo y la competitividad: al moderno contrato sociosexual. Recordemos que en el trabajo de Ubillos et al., (2000) la dimensión MAS correlacionaba negativamente con la mayor frecuencia de relaciones sexuales, por lo que no es de extrañar que en el caso de la asexualidad, la relación sea, por el contrario, positiva. Japón, un país que no ha podido ser incluido en el estudio, al no formar parte de la comunidad AVEN, pero cuyo número de hombres y mujeres que no desean relaciones sexuales es el más alto de todos, presenta, como no podía ser de otro modo, también la más alta puntuación en esta dimensión (MAS = 95) (Hofstede et al., 2010: 141).

En nuestro estudio, la correlación entre el índice MAS y el número de integrantes de cada país de la comunidad asexual (AVEN) es $r=0.61$ (Tabla 2). Tan sólo hay dos países donde la cifra es más baja de lo que cabría esperar (Polonia e Italia) y otros dos donde, por el contrario resulta más elevada de lo esperado (Países Bajos y Finlandia). Las puntuaciones de dichos países en el índice de aversión a la incertidumbre (UAI) son las que explican tales desviaciones de los valores esperados: los Países Bajos han sido tradicionalmente reconocidos por la laxitud de sus normas en diferentes materias, como la sexual, y su puntuación en el índice UAI (53) al igual que el de Finlandia (59) es inferior a la media. Por el contrario, Polonia o Italia han sido países en los que la religión ha estado relativamente más presente que en otros, y sus índices UAI son superiores a la media (93 y 75 puntos, respectivamente). Por ello, si junto al caso más extremo (Portugal, con un índice de aversión a la 
incertidumbre UAI= 104) dejáramos a un lado dichos países, observaríamos que, para el resto de países considerados, la relación entre las tasas de integrantes de la comunidad asexual y la dimensión MAS es de $r=99\left(\mathrm{R}^{2}=0.98\right)($ Gráfico 1).

La forma en la que hemos visto que el índice UAI puede influir en los resultados de la dimensión MAS, hace que la inclusión del análisis de los datos obtenidos a través de la plataforma REVA (el área de habla hispana) revista un interés especial, a pesar de las dificultades metodológicas señaladas (muestra autoseleccionada). Y ello es así en tanto que se trata de países que comparten determinadas características culturales, la más importante de las cuales consiste precisamente en su gran homogeneidad respecto al índice UAI (todos ellos se sitúan en el rango entre los 80 y los 90 puntos), por lo que las relaciones entre el peso de cada nacionalidad en la muestra y otras dimensiones culturales tal vez puedan hacerse más visibles.

En efecto, así es: en el caso de REVA, la correlación entre el número de integrantes y la dimensión MAS es $\mathrm{r}=0,65$ (Tabal 3). Pero junto a dicha dimensión, aparece ahora la importancia del individualismo $(\mathrm{r}=0,61)$, siendo los dos únicos factores que alcanzan un $\mathrm{R}^{2}$ superior al que se considera significativo (es decir, mayor de 0,30). A un menor nivel de significación, la dimensión de la permisividad muestra también una cierta correlación positiva $(\mathrm{IVR}=0,42)$, lo que podría interpretarse en el sentido de que, en el área de habla hispana, la forma en la que se vive la identidad asexual parece tener algo más que ver con la importancia de la sociabilidad, el tiempo libre y los amigos que en el caso de los países incluidos en el estudio de AVEN. Pero frente a tales dimensiones, ni la mayor cultura igualitaria, ni la orientación al largo o al corto plazo, muestran una correlación significativa con el mayor o menor número de integrantes de la comunidad asexual.

Los resultados relacionados con la dimensión UAI merecen mayor detalle, pues si de un lado, como hemos visto, puede influir en el grado en el que pueden hacerse visibles las correlaciones de otras dimensiones culturales con la comunidad asexual (como la dimensión MAS) de otra parte los resultados no muestran una relación directa con el mayor o menor número de integrantes. Ello es así porque se trata de comunidades virtuales, en las que los participantes son libres de integrarse y de expresar su identidad, sin necesidad de "salir del armario" (en la jerga asexual, sin necesidad de "bajar del ático") en otros ámbitos como el laboral, el familiar o entre las amistades. Por lo tanto, las posibilidades de ver reflejada la influencia de la dimensión UAI en el espacio virtual quedarán siempre seriamente limitadas. Las redes sociales parecen configurarse, cada vez más, como nuevos espacios de libertad en los que poder vivir ignorando algunas normatividades, del mismo modo en el que en su día lo fueron las nuevas ciudades. 
b) Actividad en los foros: la autorrealización personal.

En este caso, al igual que en el apartado siguiente, sólo se consideran los datos de AVEN (no se dispone de datos para el caso de REVA). Los resultados pueden resumirse diciendo que es el individualismo, como idea que fomenta la autorrealización y la proactividad, la dimensión que resulta más relevante cuando se miden, justamente, las tasas de actividad, mostrando una correlación positiva, tanto en el caso del número total de mensajes en los foros de cada plataforma $(r=0,51)$ como en el caso de la variable "número de mensajes por individuo" ( $\mathrm{r}=0,31)$, aunque en ninguno de los dos casos se alcance el nivel de significación, ni para el individualismo ni para ninguna otra de las dimensiones culturales (Tabla 4).

c) Crecimiento en el último año: el hoy y no el mañana.

En cuanto al crecimiento de las plataformas a lo largo de 2013 (un nada despreciable $28,54 \%$ ), los resultados muestran una correlación negativa con la orientación al largo plazo $(r=-0.59)$ lo que significa que es positiva respecto al cortoplacismo. Por lo tanto, para quienes se han integrado durante el último año, la pertenencia a la comunidad virtual cumple, prioritariamente, una función expresiva más que pragmática u orientada a la defensa de unos intereses concretos a largo o medio plazo.

Por otro lado, también se observa una fuerte correlación negativa entre el incremento de participantes y el índice de individualismo ( $\mathrm{r}=-0.8)$ lo que implica que es positiva respecto a su opuesto, el colectivismo. Sin embargo, hay que decir que, de nuevo, nos encontramos con los datos de Portugal como un caso particular y extremo, ya que su número de integrantes ha aumentado durante el último año un $133 \%$ afectando a los resultados. Si dejamos a un lado este caso extremo, vemos que la correlación entre colectivismo y crecimiento en los foros disminuye hasta un $\mathrm{r}=0.3 \mathrm{~m}$ por lo que no podemos concluir que exista una relación significativa, mientras que en el caso de la dimensión LTO (orientación al largo plazo) dicha relación no sólo mantiene su signo negativo, sino que aumenta hasta un $r=-0.63$. (Tabla 5). Ello tal vez podría estar indicando una falta de confianza en el futuro, y en ese sentido podría ser oportuno señalar que en el conjunto de los países AVEN se han encontrado correlaciones significativas entre la mayor presencia de AVEN en cada país y otros indicadores como sus respectivas tasas de fertilidad y natalidad $(r=-0,57$ y $\mathrm{r}=-0,56)$. Pero, en cualquier caso, el hecho de que sea en las sociedades más cortoplacistas donde más ha aumentado el número de integrantes, debe interpretarse como una señal de que el valor actual de pertenecer a la comunidad asexual es: 
- Lo suficientemente significativo como para despertar ese deseo de pertenencia, y

- Que es significativo en relación con el hoy y no con el mañana. Con un aquí y ahora marcado por las características de la globalización económica, los avances científico-tecnológicos y la cultura de la hiper-competitividad.

En definitiva, y como conclusión a todo lo anterior, podemos decir:

a. Que la dimensión de la masculinidad (MAS) es la que se encuentra más comprometida en la relación entre cultura e identidad asexual. Y a partir de aquí (pero sin salir de aquí, esto es, de la dimensión de la masculinidad):

b. Mayores niveles de individualismo y la existencia de normas orientadas a la autorrealización (IDV) posibilitarán a los individuos la expresión de sus preferencias sexuales, incluidas todas aquellas que se aglutinan bajo el paraguas de la asexualidad.

c. Por el contrario, mayores índices de aversión a la incertidumbre (UAI) implicarán normas más rígidas y mayores presiones para integrarse en una sexualidad normativizada, o al menos para esconder orientaciones sexuales alternativas, incluida la orientación asexual. No obstante, al tratarse esta última de una identidad construida en red (de la que no todos sus portadores hablan siempre después en el ámbito de las redes primarias o secundarias) el efecto de esta dimensión puede verse limitado. En nuestro caso, sólo ha salido a la luz cuando ha sido puesta en relación con otras variables, como la dimensión MAS.

d. La mayor cultura igualitaria, y con ella un hipotético sentimiento de desplazamiento del hombre, en lo que al ámbito de la sexualidad se refiere, no parece tener mucho que ver, ni positiva ni negativamente, con el desarrollo de la identidad asexual.

En realidad, más que a una cuestión de igualdad y de disolución actual de las diferencias, la mayor importancia del índice de masculinización social apunta, justamente, a la pervivencia de la división entre las esferas, roles y valores tradicionalmente considerados "masculinos" y "femeninos" como parte del enigma a resolver: nuestra pregunta inicial, que fue ¿cómo se hace posible que la asexualidad emerja como movimiento en pos de una identidad colectiva, en el seno de unas sociedades tan masculinizadas como Japón, Alemania o EEUU? parece poder responderse del siguiente modo: precisamente, porque lo son.

En primer lugar, y afectando al nivel micro-sociológico, la dimensión de la masculinidad es una dimensión cultural diferenciadora, clasificadora: la que más afecta al "deber ser" diferenciado (la identidad), necesitando categorías y explicaciones para una realidad humana que es fluida y que es diversa. Cuanto mayores sean los índices de masculinización social, mayores serán las presiones que perciban los individuos para explicarse y para etiquetarse, es decir, para elaborar una identidad relativa a su sexualidad, produciéndose mayores tensiones (burlas, incredulidad, desprecio) ante comportamientos considerados "poco" sexuales, lo que resulta coherente con las descripciones, tanto académicas 
(Carrigan, 2011) como autobiográficas (Lietsi, 2012), de los itinerarios vitales que suelen recorrer las personas que finalmente se auto-definen como asexuales.

En segundo lugar, la dimensión de la masculinidad es también separadora: diferencia para separar. En el contrato moderno, lo que separa es el ámbito laboral del ámbito familiar, convirtiendo en "guerra de géneros" lo que no es sino incompatibilidad socialmente construida entre la esfera pública y la privada, pero también, en el ámbito más íntimo, disocia la sexualidad del mundo de los afectos: arranca la sexualidad de la privacidad y la lleva a su terreno, transformándola en cuestión pública, social. Por tanto, es más probable que la contestación también alcance al debate público, colectivo. Son los países más masculinizados, y por tanto más sexualizados, aquellos en los que encontramos un mayor número de integrantes de la comunidad asexual.

En tercer lugar, la masculinidad es jerarquizadora: separa para jerarquizar. Al nivel del contexto más general, lo que prioriza es la esfera productiva sobre la reproductiva. La masculinización social apunta a las cada vez más crecientes exigencias del mercado laboral, tanto para los hombres como para las mujeres, a expensas de las raíces, de la familia, de la amistad o de los afectos. Todo ello explicaría, a su vez, la coincidencia en el tiempo del surgimiento de los diferentes fenómenos de desafección de la sexualidad con el cuestionamiento actual de los estilos modernos (e hipermodernos) de producción y consumo (Eguizábal, 2006; Taberna, 2012) junto a sus correlatos políticos. Los países más masculinos son aquellos en los que mayores son las tensiones entre los diferentes espacios -con diferentes conjuntos de reglas y de valores- por los que un mismo ser humano necesita transitar en su cotidianeidad.

Por lo tanto, si en el marco de la acción humana como acción simbólica nos preguntamos qué es exactamente aquello a lo que se dice "no" cuando se está diciendo "no" al sexo, la respuesta habrá de encontrarse profundizando, más que en ninguna otra parte, en el análisis de la dimensión de la masculinidad. Podríamos decir que el índice IDV muestra el grado en el que algo que se quiere individualmente se puede hacer, es decir, se hace posible y socialmente aceptable, mientras la relación de la comunidad asexual con el índice MAS habría de explicar, más bien, por qué se quiere lo que se quiere, qué valor simbólico tiene, qué significa participar, hoy y aquí, de la identidad asexual.

La principal diferencia entre la dimensión MAS y cualquier otra de las expuestas es que lo que mide no son las desigualdades, la autonomía o la incomunicación entre los seres humanos, sino el grado en el que dos conjuntos de valores distintos se mantienen o no separados (Hofstede et al., 1998). Las sociedades insisten en hiper-modernizarse (hiper-masculinizarse, hipercompetitivizarse) creando una tensión cada vez mayor entre ambas esferas de la experiencia: una tensión que no puede dejar de crear resistencias. En aquellos países en los que aquellas tensiones existen en menor grado (Noruega, Suecia) el colectivo asexual tiene también mucho menor eco. Lo que mide, por tanto, el índice MAS, no es otra cosa que la salud del moderno contrato social, que siempre fue socio-sexual (Pateman, 1995).

Sólo en virtud de todo ello, se entiende mejor que el desencantamiento y la 
decepción por un determinado orden social se manifieste en términos que son a la vez políticos, éticos, religiosos y sexuales, y todos ellos como términos en los que se expresa una misma identidad:

"Todos los humanos tenemos la capacidad de elegir nuestro camino. El mío es el de Anarquismo, Escepticismo, Ateísmo y Asexualidad." 19

En la última página de El contrato sexual, Pateman advierte que escribe en un momento en el que el contrato social ha perdido muchas de sus virtudes, de modo que si las relaciones fraternales entre los hombres se han transformado, las relaciones entre mujeres y hombres y los significados de la masculinidad, la femineidad y la propia sexualidad también podrían llegar a cambiar.

Se transforman por tanto las identidades, los modelos de relaciones y las solidaridades. Y en ese camino, el papel de las tecnologías de la comunicación y las redes sociales están mostrando su potencial, no sólo porque posibilitan el contacto entre personas que no habrían tenido posibilidad de reunirse de otro modo, sino porque constituyen nuevos espacios para la re-definición de la realidad.

\section{LA RECONSTRUCCIÓN DEL SUJETO EN EL ESPACIO VIRTUAL.}

Se imponen aires de cambio, que no afectan únicamente al terreno público: "La verdadera fuerza de este impulso está expandida muy difusamente en el cuerpo de la sociedad, en la vida cotidiana y muchas veces en la dimensión festiva de esta última, donde lo imaginario ha dado refugio a lo político y donde esta actitud es omnipresente. La impugnación o el descontento se están dando en los usos, costumbres y comportamientos, y apuntan en una dirección por lo pronto тиy росо "política"; brotan en muchos sentidos disímbolos (...) como una resistencia y una rebelión inalcanzables por el poder establecido, dirigidas a corroerlo sistemáticamente a fin de provocar en él una especie de implosión" 20 .

La forma cultural que toma este nuevo espíritu es la forma del ethos barroco, el más interesante de los cuatro ethé de Echeverría, que no son sino las formas culturales con las que los individuos se enfrentan a las contradicciones de las sociedades actuales. En palabras de Gandler, cuatro "formas de vivir lo invivible" (Gandler, 2012).

La primera de ellas es el cinismo, la negación de la existencia de las contradicciones: esta es la forma del ethos realista, que se identifica y se adhiere vorazmente al sistema. Un comportamiento que se vuelve predominante al final del siglo XX, y cuyo objetivo es subsumir cualquier aspecto cualitativo en el cuantitativo: la desaparición de la forma natural de la vida, bajo la forma

19 "Anarkopunk". www.asexuality.org/sp. Acceso 22 de noviembre de 2012

${ }^{20}$ Echeverría, en entrevista en Periódico Diagonal, el jueves, 4 de octubre de 2007. http:// www.diagonalperiodico.net/El-descontento-se-esta-dando-en.html 
(naturalizada) del mercado. Por su parte, el ethos romántico tampoco vive la contradicción porque cree falsamente que, a pesar de todo, la vida económica se organiza para satisfacer las necesidades reales de los individuos. El ethos clásico, o neoclásico, intenta vivir la realidad moderna en su día a día, con una actitud distanciada, neutral. Se ahorra el cinismo y la ingenuidad, porque no niega las contradicciones, pero las vive como algo insuperable, como una fuerza natural inevitable o como un designio divino: no aspira a luchar contra ellas, sino a elegir, en cada momento, el menor de los males.

El ethos barroco, sin embargo, va un paso más allá: tampoco niega las contradicciones y las acepta como inevitables, pero aún así, insiste en resistirse a ellas. No intenta luchar por otro mundo posible sino que actúa como si ese otro mundo ya tuviera, de facto, existencia: entre la gente corriente, entre los ejércitos de perdedores. En lo que todos los demás entienden como el mundo verdaderamente existente, el ethos barroco descubre lo que en realidad tiene de puesta en escena, a la que opone su propia capacidad representativa, performativa. El ethos barroco enfrenta las contradicciones intentando desrealizarlas, que es, tal vez, la única forma de trascenderlas:

“(...) Conviene por ello perderle el respeto a lo fáctico; dudar de la racionalidad que se inclina ante el mundo "realmente existente", no sólo como ante el mejor (dada su realidad) sino como ante el único mundo posible, y confiar en otra, menos "realista" y oficiosa, que no esté reñida con la libertad. Mostrar que lo que es no tiene más "derecho a ser" que lo que no fue pero pudo ser; que, por debajo del proyecto establecido de modernidad, las oportunidades para un proyecto alternativo -más adecuado a las posibilidades de afirmación total de la vida, que ella tiene en su esencia- no se han agotado todavía" (Echeverría, 2011).

El ethos barroco sabe, en definitiva, que el principio de realidad alcanza siempre formas históricas y contingentes. Y sabe que su contraparte, el principio del placer, también. Por eso es capaz de adentrarse en saberes y placeres poco previstos, poco pensados en un momento y lugar determinados.

Pocos espacios pueden existir actualmente, con la misma capacidad de asimilarse a ese extra-mundo oficioso, buscado, necesitado para la construcción de realidades alternativas, como el espacio virtual. Ese espacio no es condición necesaria ni suficiente para el advenimiento del ethos barroco, pero amplifica, difunde y diversifica su espíritu, no sólo por las posibilidades que ofrecen su inmaterialidad, inmediatez e interactividad, como atributos básicos de las redes sociales virtuales (Torres Albero, 2012), sino porque las ideas de autocomunicación y auto-gestión que suscitan, fomentan la sensación colectiva de que desde este extra-mundo, desde esa oficiosidad, cualquier cosa, incluida la redefinición de la realidad, es posible y que, siendo posible, debe hacerse.

En las redes virtuales se transforma la realidad, se transforman las relaciones y se transforma el sujeto, porque si la subjetividad se construye a través de la interacción (esto es, si el ser humano sólo se construye como tal en relación con otros seres humanos en un medio intersubjetivo de interacción (Honneth, 1997), las redes virtuales ofrecen la oportunidad para nuevas subjetividades, 
construidas a partir de un tipo de interacción -y de reconocimiento- diferentes, en tanto que no mediados, no ya sólo por las condiciones socio-económicas personales (que, en todo caso, tendrán siempre que alcanzar un mínimo común de recursos suficientes para sufragar los gastos de la conectividad) o no sólo por su horizontalidad (la supuesta no jerarquización de roles) sino, aún dentro de las relaciones horizontales, porque se trata de una interacción no constreñida por los sistemas normativos de la reciprocidad y del reconocimiento habituales. En la vida offline, no se habla igual con el jefe que con el compañero de trabajo; pero aún en las relaciones no jerarquizadas, tampoco se habla igual con el compañero de trabajo que con un hermano, con la pareja o con un amigo; ni se habla igual con un policía, un profesor, un médico o un bombero, aunque todos ellos sean representantes del servicio público. En la vida online, la comunicación puede ser más libre, no ya de circunstancias sociales, sino de "los límites de esos lazos previos de relaciones y sus normatividades". El quién-eres-tú habermasiano del habla desaparece (Honneth, en Pereira, 2010) y, con ello, la propia acción comunicativa se transforma.

Y aún así, desapareciendo, el sujeto tal vez puede ser más sí mismo que nunca. En las redes virtuales, el sujeto sólo es su imaginación y sus argumentos: su discurso. Pero ese discurso, libre de ese haz normativo, una vez inmaterializado y descorporeizado, en relación con la vida offline deja de ser un discurso radicalmente "situado y encarnado". El sujeto puede decir lo que quiera, lo que también puede funcionar a la inversa: que pudiendo decir, aprenda, descubra, qué es lo que quiere. Y parte de qué es eso que se quiere, es lo que se está decidiendo, deliberando, hoy en las redes. Y se está decidiendo de una forma no situada, no encarnada (si así se quiere, imaginada) que sin embargo más tarde hibrida, coloniza, la realidad offline,

En las páginas de AVEN, podemos leer que su fundación se debió en parte a las tensiones existentes entre una concepción de la asexualidad marcadamente anti-sexual y, tal vez, demasiado estrecha, mantenida anteriormente por algunos colectivos, y los grupos de asexuales queer o sex-positiv quienes, junto a otros activistas de los movimientos LGBTTIQ, habían abogado por un enfoque centrado en el apoyo mutuo ${ }^{21}$ y la construcción de un movimiento social, en el

21 No se ha hecho referencia en estas páginas a las tensiones que, al margen de acuerdos teóricos bienintencionados, existen en la realidad cotidiana de los diferentes grupos de intimidad, en relación a las dificultades para su pleno reconocimiento mutuo, tal y como ilustra, por ejemplo, Nieto (2003) en el caso de la relación entre transexuales y transgeneristas no transexuales, y tal y como puede advertirse en algunos de los comentarios presentes en los foros de AVEN, para el caso, también por ejemplo, de la relación entre la asexualidad y la queerness (www.asexuality.org/sp). Las políticas de identidad se encuentran en la realidad preñadas, no sólo del deseo de contestación a la normatividad sexual dominante, sino también de los miedos de cada cuál a que los intereses propios (cuya defensa suele haber costado, como mínimo, sudores durante largo tiempo) queden subsumidos y anulados en la pluralidad de voces que defienden, a la vez, los suyos. Como si no fueran los mismos. Como si no fuera uno sólo. De ese modo, las políticas de identidad se transforman, ellas mismas, en esencializadoras, suprimen unas diferencias pero crean otras, tal y como advertía Plummer (2003:82) y tal y como la propia firmante de este trabajo comprende bien, si ha de ser sincera consigo misma, recordando sus miedos propios en tanto que heterosexual feminista 
que:

a) La asexualidad fuese entendida como una identidad flexible que las personas debían definir por sí mismas y que debía servir como "herramienta" y no como "etiqueta" y

b) El proyecto fuera definido como "espacio de apoyo donde las personas asexuales pudieran explorarse a sí mismas y, finalmente, auto-organizarse en torno a la visibildiad y el trabajo de educación" 22 .

Se observa aquí la concepción de la re-construcción del sujeto como ente / proceso dinámico, poroso, interactivo. En el segundo volumen de La era de la información, Castells señala que "las identidades que expresan proyectos de identidad orientados a cambiar los códigos culturales deben ser movilizadoras de símbolos. Han de actuar sobre la cultura de la virtualidad real que encuadra la comunicación en la sociedad red, subvirtiéndola en nombre de valores alternativos e introduciendo códigos que surgen de proyectos de identidad autónomos” (Castells, M., 2003 [1997], 464). En este sentido, resulta difícil imaginar un disímbolo mayor para nuestros días que la a/anti/contra-sexualidad (Preciado, 2011).

El espacio virtual es una herramienta privilegiada que está ayudando a algunos a hablar consigo mismos, pero que también puede ayudar a hablar con los otros. Si recordamos la caracterización de las sociedades actuales como sociedades centrífugas (en oposición a la modernización centrípeta), podríamos decir que puede ayudar a las periferias a conectarse entre sí, sin depender y sin necesidad de dirigirse a un centro. Pero, precisamente por ello, lo paradójico de las potencialidades de la situación es que, frente al temor de una parte del discurso asexual (a quedar subsumido en el océano de la multiplicidad de voces e identidades sexuales actuales), sólo fundiéndose en esa corriente, sólo en esa confluencia de todos con todos (online y offline), pueden lo fenómenos minoritarios transformarse en nuevo significante y alcanzar un sentido social real específico y sólo así puede dotarse cada uno de ellos de un significado reconocible por todos. Hoy la agenda social no es (o al menos no es tan sólo) política: es una multi-conferencia, una agenda re-codificadora, cultural, interpersonal. Menos que nunca, una identidad (o campo de identificación) debería temer hoy a los otros, porque tal vez sólo en el contexto que conforman todos ellos, son susceptibles de adquirir significado -y respeto y reconocimiento recíproco- propios.

que no querría ver sus expectativas frustradas, inmersas en la misma multiplicidad y diversidad de necesidades y voces. Tenemos que hablarnos más.

22 http://www.asexuality.org/wiki/index.php?title=Haven_for_the_Human_Amoeba 


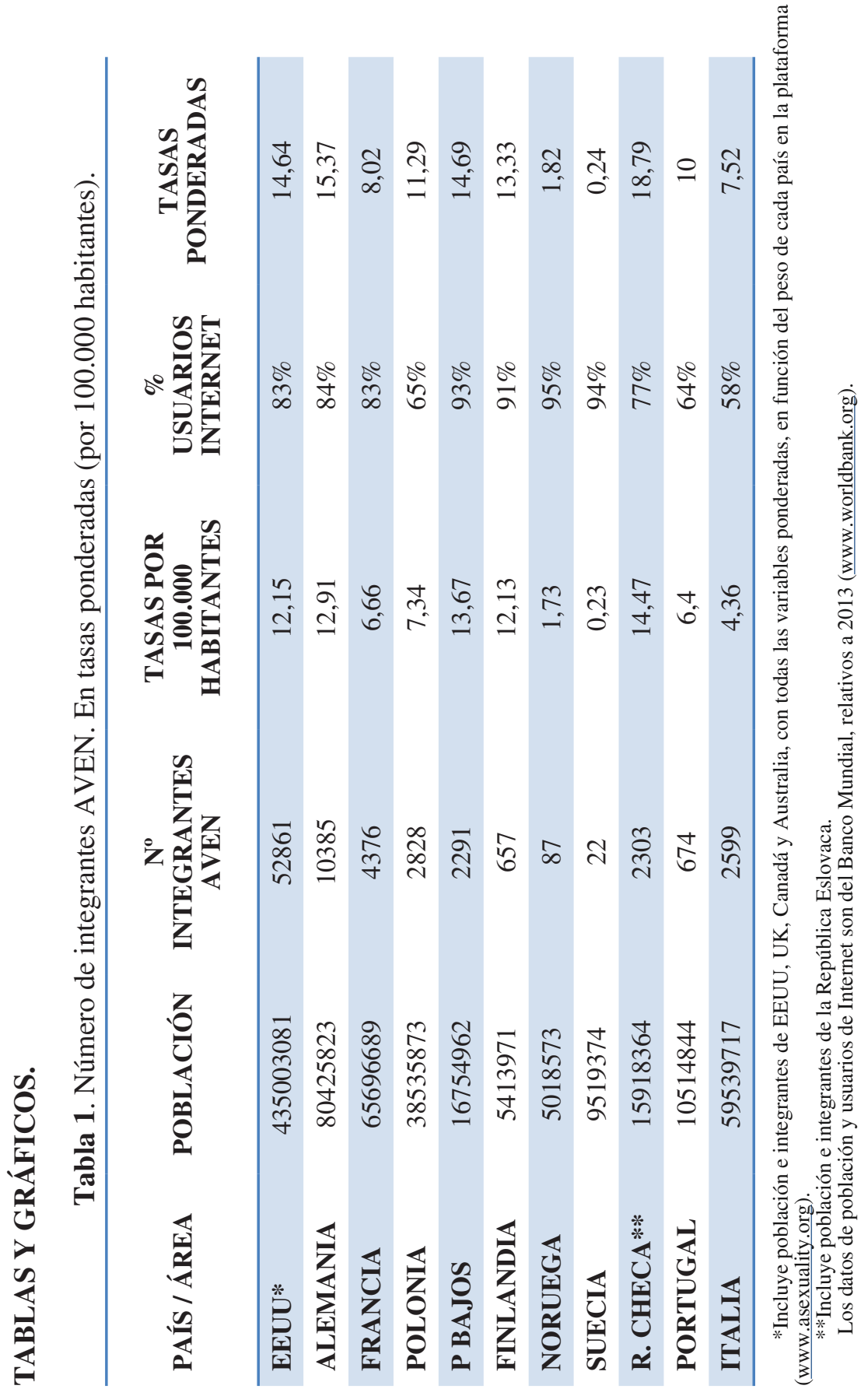

EMPIRIA. Revista de Metodología de Ciencias Sociales. N. ${ }^{\circ}$ 32, septiembre-diciembre, 2015, pp. 129-168. ISSN: 1139-5737, DOI/empiria. 32.2015.15312 


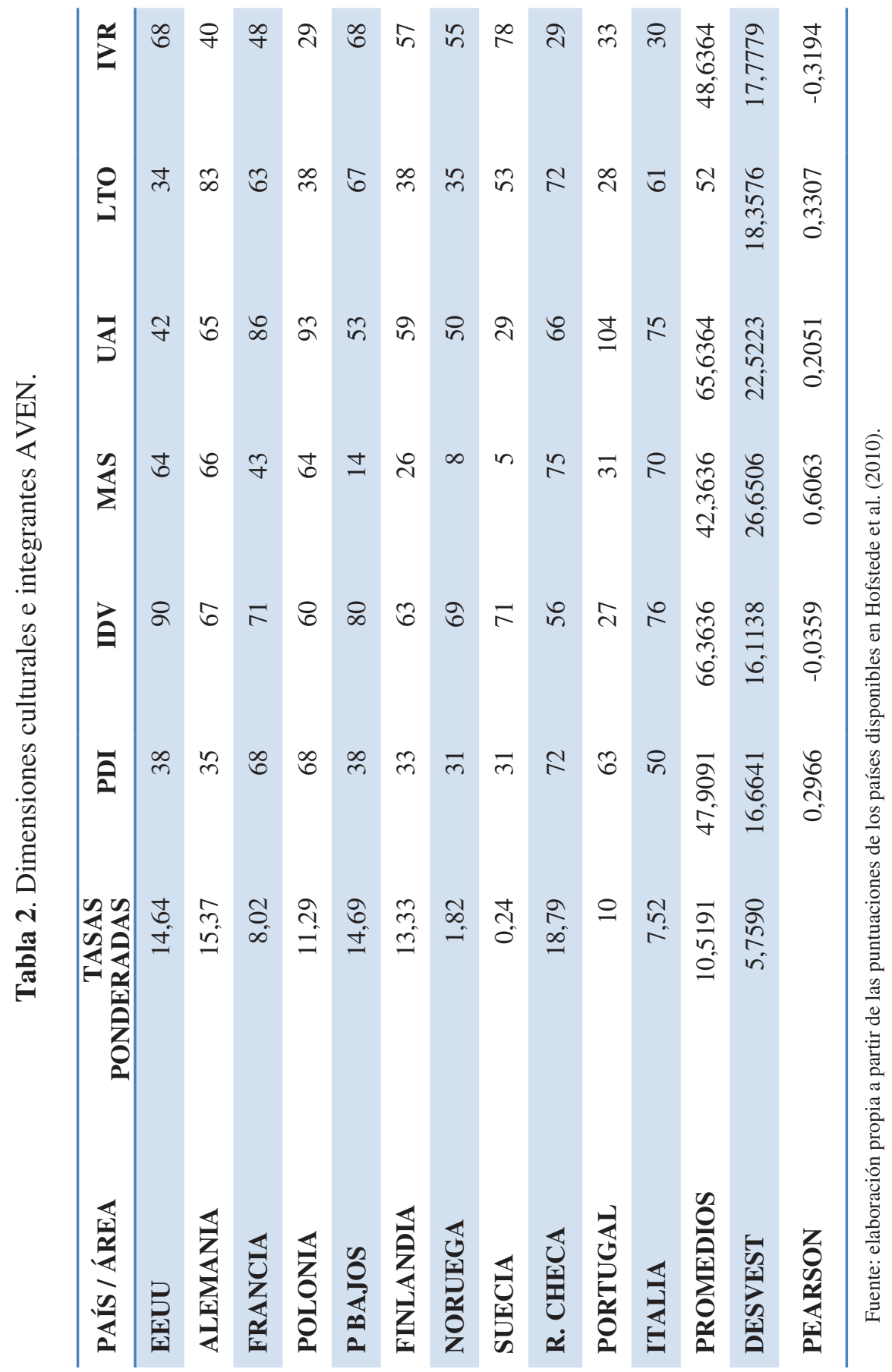

EMPIRIA. Revista de Metodología de Ciencias Sociales. N. o 32, septiembre-diciembre, 2015, pp. 129-168. ISSN: 1139-5737, DOI/empiria. 32.2015.15312 


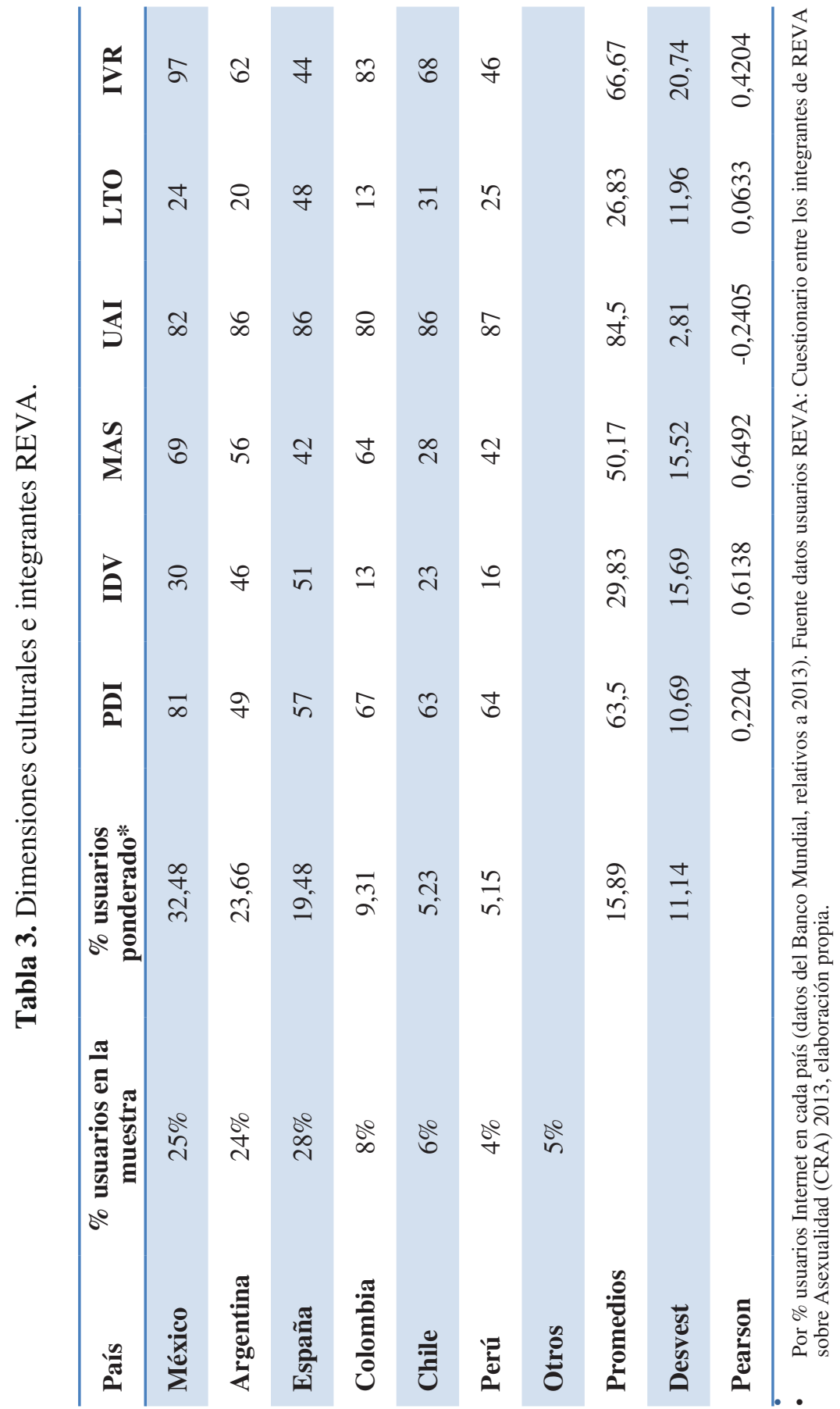

EMPIRIA. Revista de Metodología de Ciencias Sociales. N. ${ }^{\circ}$ 32, septiembre-diciembre, 2015, pp. 129-168. ISSN: 1139-5737, DOI/empiria. 32.2015.15312 


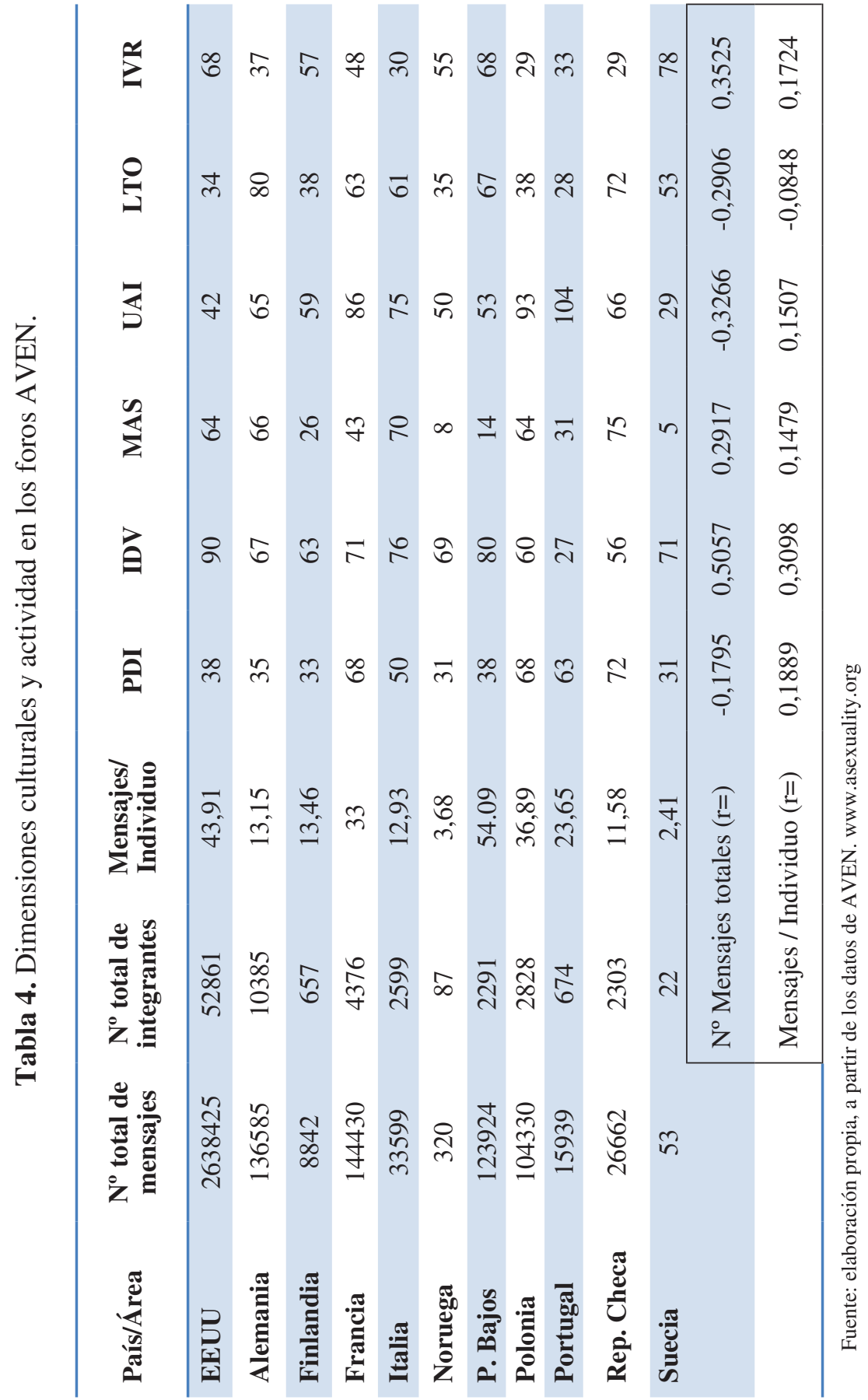

EMPIRIA. Revista de Metodología de Ciencias Sociales. N. ${ }^{\circ}$ 32, septiembre-diciembre, 2015, pp. 129-168. ISSN: 1139-5737, DOI/empiria. 32.2015.15312 


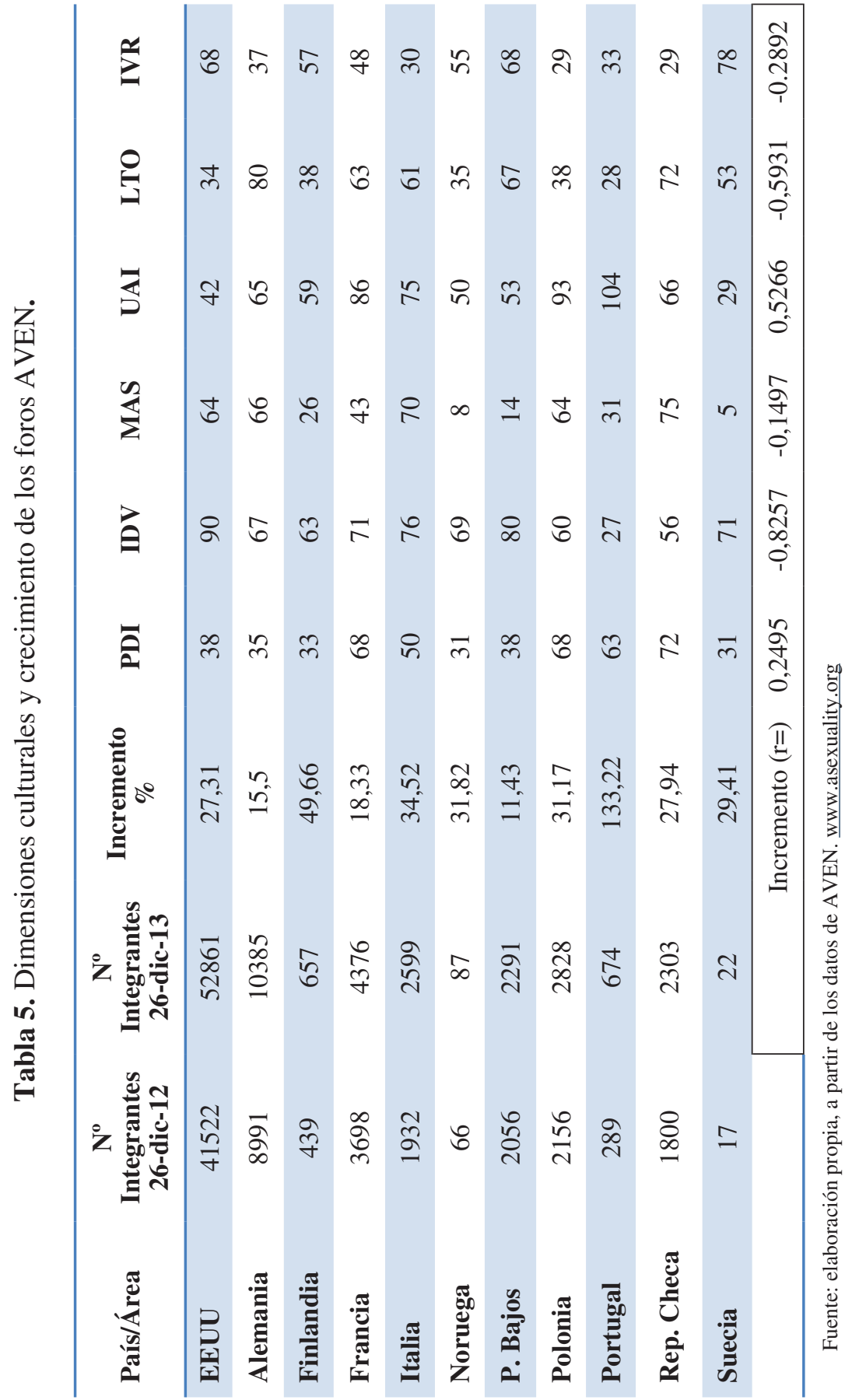

EMPIRIA. Revista de Metodología de Ciencias Sociales. N. ${ }^{\circ}$ 32, septiembre-diciembre, 2015, pp. 129-168. ISSN: 1139-5737, DOI/empiria. 32.2015.15312 




EMPIRIA. Revista de Metodología de Ciencias Sociales. N. ${ }^{\circ}$ 32, septiembre-diciembre, 2015, pp. 129-168. ISSN: 1139-5737, DOI/empiria. 32.2015.15312 


\section{Gráfico 1. Correlación entre el índice MAS y las tasas de integrantes AVEN (países núcleo MAS/FEM, por 100.000 habitantes)}

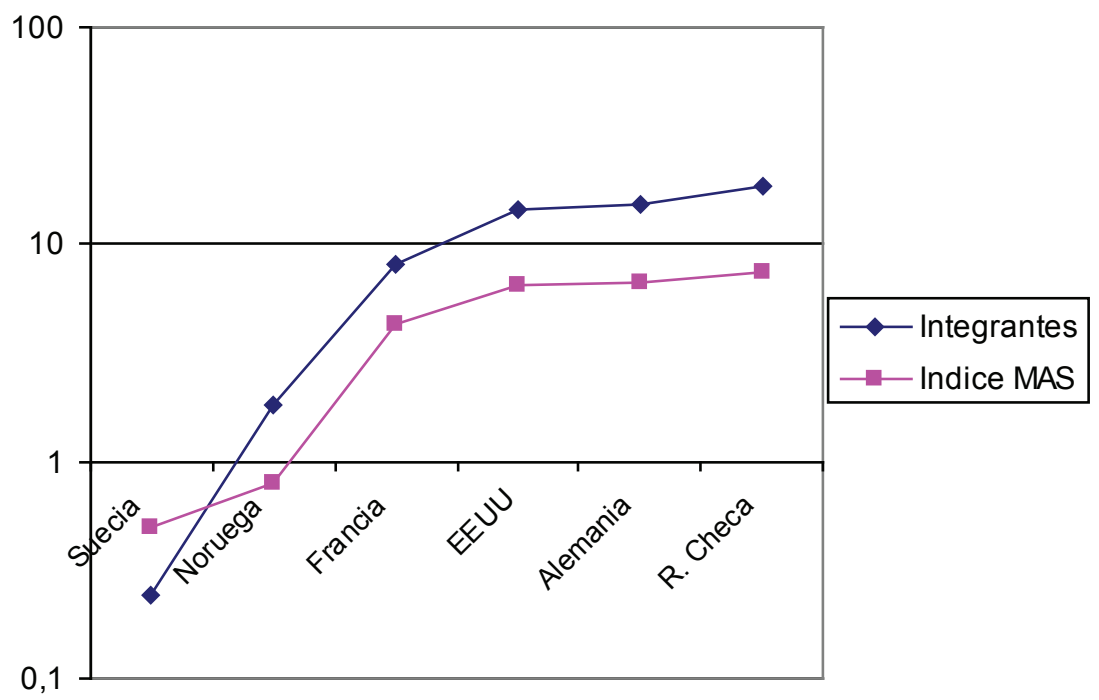

\section{BIBLIOGRAFÍA}

AGUINAGA ROUSTÁN, J. (2004): El precio de un hijo: los dilemas de la maternidad en una sociedad desigual. Madrid, Debate.

ANDUIZA, E., CANTIJOCH, M., GALLEGO, A. Y SALCEDO,J. (2010): Internet y participación política en España, Madrid, Centro de Investigaciones Sociológicas, Colección "Opiniones y Actitudes", nº 63.

BAUMAN, Z. (2005): Amor líquido. Acerca de la fragilidad de los vínculos humanos. Barcelona, Fondo de Cultura Económica de España.

BECK, U. (2009): La sociedad del riesgo global. Madrid, Siglo XXI.

BECK, U., BECK-GERNSHEIM, E. (2001): El normal caos del amor. Las nuevas formas de la relación amorosa. Barcelona, Paidós - El Roure.

BOGAERT, A. (2012): Understanding asexuality, UK, Rowman Littlefield Publishers, Inc.

BORDIEU, P.(1997): Razones prácticas. Sobre la teoría de la acción. Barcelona, Anagrama, 
BUTLER, J. (2002): Cuerpos que importan. Sobre los límites y materiales discursivos del "sexo". Buenos Aires, Paidós SAICF.

BUTLER, J. (2007): El género en disputa. El feminismo y la subversión de la identidad. Barcelona, Paidós.

CABAÑES, E (2012) 01: "De la hibridación al procomún, construyendo la realidad a través de la tecnología", Jóvenes, tecnofilosofía y arte digital, Revista de Estudios de Juventud, no 102, septiembre 2012, INJUVE.

CARRIGAN, M. (2011): "There's more to life than sex? Difference and commonality within the asexual community". Sexualities, 14, 462-478.

CASTELLS, M. (2003): La era de la información, Vol. 2, El poder de la identidad. Madrid, Alianza Editorial.

CHARLEBOIS, J. (2013): "Herbivore masculinity as an oppositional form of masculinity". Culture, society \& masculinities, Vol. 5 issue 1, spring 2013, pp. 89-104. The man's studies press, Ilc.

EGUIZABAL, R. (2006): "El postconsumo. La condición consumidora en la Sociedad de la Información", Telos, Cuadernos de comunicación e innovación. № 67, 2006, 35-44.

ECHEVERRÍA, B. (Antología) (2011): Crítica de la modernidad capitalista. Vicepresidencia del Estado Plurinacional de Bolivia. La Paz.

ECHEVERRÍA, J. ( 2008): 06: “Cuerpo electrónico e identidad".1. 1 Este artículo ha sido elaborado en el marco del proyecto de investigación sobre "Axiología y dinámica de la tecnociencia" financiado por el ministerio de Ciencia y Tecnología durante el período 2000-2002. Cuerpo-Experimental-Transmutativo. Centro Multimedia del CENART, México.

FREITAS, D. (2013): The end of sex: how hookup culture is leaving a generation unhappy, sexually unfulfilled and confused about intimacy. New York. Basic Books, A member of the Perseus Books Group.

GANDLER, S. (2012): "Reconocimiento versus Ethos". Iconos. Revista de Ciencias Sociales. No 43, Quito, Mayo 2012, pp. 47-64. Facultad Latinoamericana de Ciencias Sociales. Sede Académica de Ecuador.

GUASCH, O. (2007): La crisis de la heterosexualidad. Barcelona, Editorial Laertes.

HOFSTEDE G. and ASSOCIATES (1998): Masculinity and femininity, the taboo dimension of national cultures. California, Thousand Oaks, Sage Publications.

HOFSTEDE, G. (2001): Culture's consequences, comparing values, behavior, institutions and organizations across nations, California, Thousand Oaks, Sage Publications.

HOFSTEDE G, HOFSTEDE G.J, MINKOV M. (2010): Cultures and organizations, software of the mind. Intercultural Cooperation and Its Importance for Survival, New York, McGraw Hill.

HONNETH, A. (1997): La lucha por el reconocimiento. Por una gramática moral de los conflictos sociales. Barcelona, Crítica.

KINSEY AC, POMERY WB \& MARTIN CE (1998 [1948]): Sexual behavior in the human male. Bloomington: Indiana University Press.

KINSEY AC, POMERY WB, MARTIN CE \& GEBHARD PH (1998[1953]): Sexual behavior in the human female. Bloomington: Indiana University Press.

LIETSI, L. (2012): Diario de una asexual. Edison Mera (ed.). Bubok Publishing, S.L.

LLEDÓ, E. (2007): "El lenguaje de la identidad". Congreso de la Fundación Caballero Bonald bajo el título "Periodismo y Literatura" celebrado en Jerez de la Frontera (Cádiz - España) 17 a 19 de octubre 2007. 
http://www.literaturas.info/Revista/2013/03/el-lenguaje-de-la-identidad-emilio-lledo/

NIETO PIÑEROBA, J.A. (Ed.) (2003): Antropología de la sexualidad y diversidad cultural. Madrid, Talasa.

NIETO PIÑEROBA, J.A. (2008): Transexualidad, intersexualidad y dualidad de género. Barcelona, Bellaterra.

OSBORNE, R. Y GUASCH, O. (comps.) (2003): Sociología de la sexualidad, Madrid, Centro de Investigaciones Sociológicas.

PATEMAN, C. (1995): El contrato sexual. Barcelona, Editorial Anthropos.

PEREIRA, G. (2010): "Reconocimiento y criterios normativos. Entrevista a Axel Honneth”. Andamios. Vol. 7, n 13. Mayo-agosto 2010. Pp. 323-334.

PLUMMER, K. (2003): Intimate citizenship, private decisions and public dialogue. University of Washington Press.

POSTON DL \& BAUMLE AK (2010): "Patterns of Asexuality in the United States". En Vaupel, J.W. (ed): Demographic Research. 2010, 23, 509-530. Max Planck Institute for Demographic Research.

PRECIADO, B. (2011): Manifiesto contrasexual. Barcelona, Anagrama.

ROSIN, H. (2012): The end of men (and the rise of women). New York, Riverhead Books.

SPENGLER (2012) (Spengler es el pseudónimo con el que firma David P. GOLDMAN): "Japan's lost libido and America's asexual future" en Asia Times Online, 13 de marzo de 2012. Disponible en http://www.atimes.com/atimes/Japan/NC13Dh01. html.

SPIEGELHALTER, D. (2015): Sex by numbers. What statistics cant tell us about sexual behavior. London, Profile Books Ltd., en asociación con Wellcome Collection.

STOLTENBERG, J (2000): Refusing to be a man, essays on sex and justice, London, UCL Press.

STORMS, M. D. (1980): "Theories of Sexual Orientation". Journal of Personality and Social Psychology Vol. 38: 783-792.American Psychological Association.

TABERNA, F. (2012): "Postconsumismo", Nuevatribuna.es, 28 de febrero de 2012. Disponible en http://www.nuevatribuna.es/opinion/felix-taberna/ postconsumismo/20120228171209071161.html

TIJDENS, K.G. Y VAN KLAVEREN, M. (2012): Detenida en el tiempo: la brecha salarial de género no ha cambiado en 10 años. Bruselas, Confederación Sindical Internacional.

TORRES ALBERO, C. (2012): "Redes sociales, nuevas identidades y conflictos sociales". XII Foro de Tendencias Sociales. UNED. 22-24 de marzo de 2012.

TOURAINE, A. (2005): Un nuevo paradigma. Para comprender el mundo de hoy. Barcelona, Paidós.

UBILLOS S, PAEZ D, GONZÁLEZ J.L. (2000): “Culture and sexual behavior” Psycothema, Vol.12, Supl, 70-82. Disponible en:

http://www.unioviedo.es/reunido/index.php/PST/article/view/7809/7673

USHIKUBO, M. (2008): The herbivorous ladylike men who are changing Japan. TokioKodansha, Infinity, 


\section{Otros recursos en red:}

- The Kinsey Institute: www.kinseyinstitute.org

- The Hofstede Centre: http://geert-hofstede.com

- Instituto Nacional de Investigación de la Población y Seguridad Social de Japón: http://www.ipss.go.jp/site-ad/index_english/Survey-e.asp.

- Asexual Visibility and Education Network (AVEN): www.asexuality.org

- Asexuality livejournal community: asexuality.livejournal.com

- Asexual Explorations: www.asexualexplorations.net

- Asexuality Studies Archives. Email discussion lists for the UK Education and Research communities: www.jiscmail.ac.uk

- Apositive: www.apositive.org

- A Proudly Geeky Asexual Mind. http://amoebageek.blogspot.com

- Asexy Beast. http://theonepercentclub.blogspot.com

- Love from the asexual underground. http://asexualunderground.blogspot.com

- Red para la Educación y la Visibilización de la Asexualidad (REVA): www. asexuality.org/sp.

- Diarios de Asexualidad: http://diariosdeasexualidad.blogspot.com.es 
